Document downloaded from:

http://hdl.handle.net/10251/155129

This paper must be cited as:

Orellana, M.; Selga, J.; Vélez, P.; Sans, M.; Rodríguez Pérez, AM.; Bonache, J.; Boria Esbert, VE.... (2017). Design of Capacitively Loaded Coupled-Line Bandpass Filters With Compact Size and Spurious Suppression. IEEE Transactions on Microwave Theory and Techniques. 65(4):1235-1248. https://doi.org/10.1109/TMTT.2016.2638843

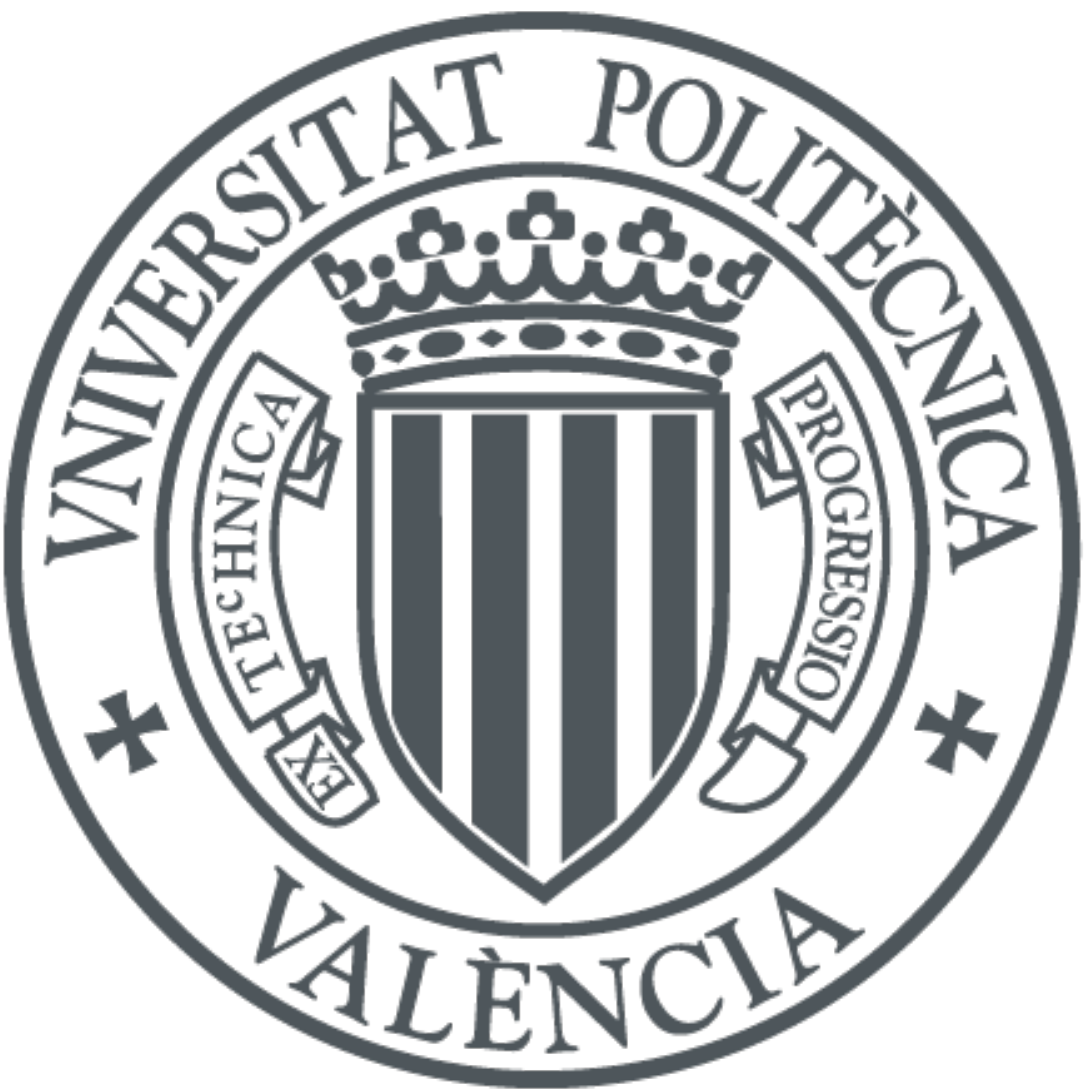

The final publication is available at

https://doi.org/10.1109/TMTT.2016.2638843

Copyright Institute of Electrical and Electronics Engineers

Additional Information 


\title{
Design of Capacitively Loaded Coupled-Line Bandpass Filters With Compact Size and Spurious Suppression
}

\author{
Marco Orellana, Jordi Selga, Member, IEEE, Paris Vélez, Member, IEEE, Marc Sans, \\ Ana Rodríguez, Member, IEEE, Jordi Bonache, Member, IEEE, \\ Vicente E. Boria, Senior Member, IEEE, and Ferran Martín, Fellow, IEEE
}

\begin{abstract}
This paper is focused on the synthesis of capacitively loaded coupled lines for the design of bandpass filters with compact size and spurious suppression. The filters consist of cascaded pairs of coupled lines periodically loaded with patch capacitors. The loading capacitances provide a slow-wave effect to the coupled lines useful for filter miniaturization, whereas periodicity introduces bandgaps that can be controlled in order to achieve spurious suppression. Filter design is achieved following an automated process based on aggressive space mapping (ASM) optimization. The two reported examples, a third-order filter with central frequency $f_{0}=1.0 \mathrm{GHz}, 8 \%$ fractional bandwidth, and 0.1-dB ripple level and a fifth-order filter with central frequency $f_{0}=1.8 \mathrm{GHz}, 8 \%$ fractional bandwidth, and $0.1-\mathrm{dB}$ ripple level, demonstrate the potential of the proposed ASM-based design approach, as well as the effectiveness of the loading capacitances to reduce filter size and reject the spurious bands. Significant size reduction in both filters is achieved, whereas the out-ofband rejection is better than 30 and $45 \mathrm{~dB}$ in the third-order and fifth-order filters, respectively, up to at least $4 f_{0}$.
\end{abstract}

Index Terms-Bandpass filters, circuit synthesis, coupled lines, electromagnetic bandgap, microstrip technology, slow-wave transmission lines, space mapping.

\section{INTRODUCTION}

$\mathbf{S}$ ZE reduction and spurious suppression have been (and are still) two challenging issues in planar microwave filter design. There are many approaches to achieve compact size and/or spurious cancellation. Among them, periodic transmission lines, including nonuniform and reactively loaded lines, have been used as building blocks for the design of

This work was supported in part by MINECO-Spain under Project TEC2013-47037-C5-1-R, Project TEC2013-40600-R, and Project TEC2013-49221-EXP, in part by the Generalitat de Catalunya under Project 2014SGR-157, and in part by FEDER funds. The work of F. Martín was supported by the Institució Catalana de Recerca i Estudis Avançats. The work of M. Orellana was supported in part by the Universidad de Costa Rica, in part by MICITT, and in part by CONICIT.

M. Orellana, J. Selga, P. Vélez, M. Sans, J. Bonache, and F. Martín are with GEMMA/CIMITEC, Departament d'Enginyeria Electrònica, Universitat Autònoma de Barcelona, 08193 Bellaterra, Spain (e-mail: ferran.martin@uab.es).

A. Rodríguez and V. E. Boria are with the Departamento de Comunicaciones-iTEAM, Universitat Politècnica de València, 46022 Valencia, Spain (e-mail: vboria@dcom.upv.es). microwave filters, where the ordinary lines are replaced with these artificial lines (see [1] and the references therein).

Periodicity gives rise to stopbands, due to the well-known Bragg effect [2], [3]. Such periodicity can typically be achieved by modulating the transverse dimensions of the line [1], [4], [5] or by etching holes (or other patterns) in the ground plane [6]-[10]. The result is a periodic variation of the line impedance (and coupling coefficient [1], [11]-[13]) that causes the appearance of stopbands useful for spurious suppression. To this end, it is necessary to tune the period of the lines in order to set the Bragg frequency, where maximum attenuation occurs, within the spurious band [4]. Multiple tuned periodic transmission lines can also be designed in order to achieve signal rejection at various (desired) frequencies [10]. Using this strategy, efficient multispurious suppression in coupled line bandpass filters has been demonstrated [14].

By modulating the transverse dimensions of the line, significant rejection (and hence efficient spurious suppression) can be obtained. However, this strategy has no impact on filter size. For size reduction and simultaneous spurious rejection, transmission-line sections with periodically loaded capacitors become a solution [15]-[18]. Periodicity is responsible for spurious elimination, as discussed before, whereas the loading capacitors enhance the effective capacitance of the line, thus reducing the phase velocity. These periodic capacitively loaded slow-wave transmission lines are therefore promising candidates for the purpose of this paper, i.e., the design of compact and spurious free bandpass filters based on coupled lines. Nevertheless, the application of these slow-wave structures to the design and implementation of other compact and spurious-suppressed filters has been demonstrated [19]-[22]. Capacitively loaded lines have also been applied to the miniaturization of couplers [23], and other periodic slow-wave structures based on inductive [24] or combined inductive/ capacitive [25]-[30] loading have been reported. The slowwave concept based on capacitive or dielectric loading has also been used for size reduction in CMOS passive and active devices operating at millimeter wavelengths [31]-[35].

In this paper, slow-wave coupled lines based on patch capacitors [36]-[39] are applied to the implementation of compact bandpass filters with wide stopband (this paper is indeed an extended version of [40]). Special emphasis is put on justifying the conditions to achieve efficient cancellation of the spurious 


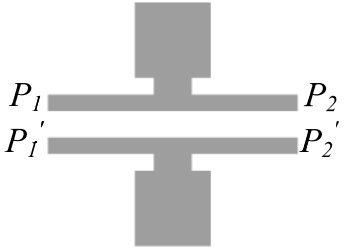

(a)

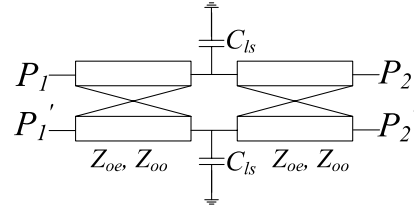

(b)

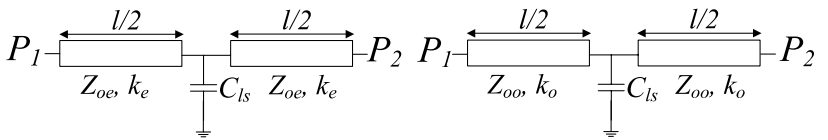

(c)
Fig. 1. (a) Typical topology (unit cell) of the CLCLs. (b) Equivalent circuit schematic. Circuit model for (c) even and (d) odd modes.

bands (related to the number of patch capacitors per coupled line section), and we present an accurate design methodology for the capacitively loaded coupled lines (CLCLs). The design approach is based on aggressive space mapping (ASM) optimization [41]-[47], where the layouts of the coupled lines are unattendedly generated from the design parameters (even/odd mode impedances and electrical length) provided by the specifications and from the required slow-wave factor (which determines the level of miniaturization of the final filter compared with the conventional counterpart).

This paper is organized as follows. In Section II, the topology of the CLCLs, the circuit model of the unit cell, and the main design equations are presented. From these equations, we are able to obtain the schematic of the coupled lines, which is the starting point for optimization using the developed ASM algorithm (presented in Section III). Two filter examples, where the synthesized coupled line sections are merely cascaded, are reported in Section IV. Finally, the main conclusions of this paper are highlighted in Section V.

\section{Topology of The ClCLs, Modeling, AND Design}

The topology and circuit schematic of the proposed CLCLs (unit cell) are depicted in Fig. 1(a) and (b), respectively. The square patches are described by the two capacitances $C_{l s}$ (note that the inductive effect of the wide and short strip connecting the patches to the coupled lines is neglected). The impedances $Z_{o e}$ and $Z_{o o}$ are the characteristic impedances of the even and odd modes, respectively, of the unloaded coupled lines, whereas $k_{e}$ and $k_{o}$ are the phase constants for such modes. Hence, the electrical lengths of the unloaded coupled lines are $\phi_{e}=k_{e} l$ (even mode) and $\phi_{o}=k_{o} l$ (odd mode), provided that $l$ is the physical length of such lines. The equivalent circuits for the even and odd modes, inferred by removing one of the halves and considering the bisection symmetry plane as a magnetic (even mode) and an electric (odd mode) wall, are depicted in Fig. 1(c) and (d). In this paper, it is assumed that the coupling between the square patches is negligible.

The dispersion relation for the even mode is given by [1]

$$
\cos \left(\beta_{e} l\right)=\cos \left(k_{e} l\right)-\frac{\omega C_{l s} Z_{o e}}{2} \sin \left(k_{e} l\right)
$$

whereas the characteristic (Bloch) impedance is

$$
Z_{B e}=\frac{Z_{o e}\left\{\sin \left(k_{e} l\right)-Z_{o e} \omega C_{l s} \sin ^{2}\left(k_{e} l / 2\right)\right\}}{\sin \left(\beta_{e} l\right)}
$$

where $\beta_{e}$ is the phase constant of the loaded coupled lines for the even mode (providing an electrical length of $\varphi_{e}=\beta_{e} l$ for that mode) and $\omega=2 \pi f$ is the angular frequency. Formally, identical expressions are obtained for the dispersion and Bloch impedance of the odd mode by simply changing the subindices

$$
\begin{aligned}
\cos \left(\beta_{o} l\right) & =\cos \left(k_{o} l\right)-\frac{\omega C_{l s} Z_{o o}}{2} \sin \left(k_{o} l\right) \\
Z_{B o} & =\frac{Z_{o o}\left\{\sin \left(k_{o} l\right)-Z_{o o} \omega C_{l s} \sin ^{2}\left(k_{o} l / 2\right)\right\}}{\sin \left(\beta_{o} l\right)} .
\end{aligned}
$$

Note that, contrary to ordinary coupled lines, these CLCLs are intrinsically dispersive and exhibit passbands and stopbands for both modes [1], [20]. In this paper, the considered CLCL-based bandpass filters will be designed to operate in the first allowed band of their constitutive CLCLs, whereas the first rejected band of the CLCLs will be tailored in order to suppress the spurious bands, unavoidable in ordinary coupled line bandpass filters. The presence of the patch capacitors modifies the coupling level of the unloaded lines, but the impedances of the even and odd modes of the loaded coupled lines, as well as the electrical length at the frequency of interest, can be adjusted to the values required by design.

An important parameter in capacitively loaded artificial lines is the slow-wave ratio, defined as the ratio between the phase velocities of the loaded and unloaded line, or equivalently, as the ratio between the phase constants of the unloaded and loaded lines. Since the phase velocities (or phase constants) for each mode (even and odd) in coupled lines are not (in general) identical (although very similar if the coupling level is small), the slow-wave ratio should be defined for each mode

$$
\begin{aligned}
& s w r_{e}=\frac{k_{e} l}{\beta_{e} l} \\
& s w r_{o}=\frac{k_{o} l}{\beta_{o} l} .
\end{aligned}
$$

\section{A. Design of the CLCLs: Determination of the Circuit Schematic}

Let us now use the previous equations for the determination of the circuit schematic of the CLCLs able to satisfy certain specifications. This means to provide the electrical parameters of the circuit of Fig. 1(b), i.e., $C_{l s}, Z_{o e}, Z_{o o}$, and either $k_{e} l$ or $k_{o} l$ (since $k_{e} l$ and $k_{o} l$ are mutually dependent through $Z_{o e}$ and $Z_{o o}$ ). With regard to the specifications, since the purpose of this paper is the design of coupled line bandpass filters, the even and odd mode impedances of the CLCLs $\left(Z_{B e}\right.$ and $\left.Z_{B o}\right)$ are dictated by filter specifications (order, fractional bandwidth, and ripple level) according to well-known formulas [48]. Note, however, that, due to dispersion, the Bloch impedances are frequency dependent, and therefore the required values of $Z_{B e}$ and $Z_{B o}$ are forced to be satisfied at the filter central frequency, $f_{0}$. Each CLCL section (composed at least of one unit cell) should exhibit an electrical 
length at $f_{0}$ of $\pi / 2$ for each mode (as corresponds to conventional parallel coupled line bandpass filters). Since this is not possible in practice (at least in microstrip technology), an alternative condition depending on these two electrical lengths is necessary. For instance, we can force the average electrical length of the CLCL section corresponding to the two modes to satisfy this requirement, or

$$
N \frac{\left(\varphi_{e}\left|f_{0}+\varphi_{o}\right|_{f_{0}}\right)}{2}=\frac{\pi}{2}
$$

where $N$ is the number of unit cells of the loaded coupled line (nevertheless, it has been found that there is a more appropriate condition, to be discussed later). Finally, a fundamental parameter that determines the level of miniaturization of the final filter is the slow-wave ratio. We will set the slow-wave ratio corresponding to the even mode $s w r_{e}$ to a predefined value. Note, however, that $s w r_{e}$ is not related to filter performance, but only to filter size. The fact that the circuit schematic of the CLCLs [Fig. 1(b)] includes an additional parameter $\left(C_{l s}\right)$, compared with ordinary coupled lines, is the key factor in having certain control on filter size reduction.

Inspection of (1)-(7) reveals that there are eight unknowns in the model, that is, $C_{l s}, Z_{o e}, Z_{o o}, k_{e} l, k_{o} l$, swr $r_{o}, \beta_{o} l$, and $\beta_{e} l\left(Z_{B e}, Z_{B o}\right.$, and $s w r_{e}$ are fixed according to the previous paragraph). However, as previously mentioned, $k_{e} l$ and $k_{o} l$ are linked through $Z_{o e}$ and $Z_{o o}$, which means that all model parameters can be univocally determined. To this end, we have proceeded as follows. We provide a guess value for $\varphi_{e}=\beta_{e} l$. From (1) and (5) evaluated at $\omega_{0}=2 \pi f_{0}$, we obtain

$$
C_{l s} Z_{o e}=\frac{2\left\{\cos \left(s w r_{e} \cdot \beta_{e} l\right)-\cos \left(\beta_{e} l\right)\right\}}{\omega_{o} \sin \left(s w r_{e} \cdot \beta_{e} l\right)}
$$

where all the parameters on the right-hand side are known. By introducing (8) in (2), we can express $Z_{o e}$ as a function of $Z_{B e}, s w r_{e}$, and $\beta_{e} l$ as

$$
Z_{o e}=\frac{Z_{B e} \cdot \sin \left(\beta_{e} l\right)}{\sin \left(s w r_{e} \cdot \beta_{e} l\right)-C_{l s} Z_{o e} \omega_{0} \sin ^{2}\left(s w r_{e} \cdot \beta_{e} l / 2\right)}
$$

provided that $C_{l s} Z_{o e}$ in the denominator of (9) is given by (8). Once $Z_{o e}$ is known, $C_{l s}$ is calculated from (8). Also note that $k_{e} l$ is known from (5). The determination of the four remaining parameters $\left(Z_{o o}, k_{o} l, s w r_{o}\right.$, and $\left.\beta_{o} l\right)$ is not simple since the relation between $k_{e} l$ and $k_{o} l$ (through $Z_{o e}$ and $Z_{o o}$ ) depends on substrate parameters. Thus, we have proceed by providing a tentative value of $s w r_{o}$, starting with $s w r_{o}=s w r_{e}$, and numerically solving (3), (4), and (6). With the resulting element values $\left(Z_{o o}, k_{o} l\right.$, and $\left.\beta_{o} l\right)$ and substrate parameters, we check if $k_{o} l$ and $k_{e} l$ are compatible. If this is not the case, we sweep $s w r_{o}$ until the resulting values of $k_{o} l$ and $k_{e} l$ are compatible with $Z_{o o}, Z_{o e}$, and the parameters of the substrate (which provide the transverse geometry of the unloaded coupled lines). We repeat this procedure until condition (7), or another equivalent condition, to be specified later, is satisfied.

Let us consider as an example the design of a pair of CLCLs with $Z_{B e}=72.12 \Omega$ and $Z_{B o}=38.91 \Omega$, swr $r_{e}=0.5$, and $\left(\varphi_{e}+\varphi_{o}\right) / 2=\pi / 4$ at $2.4 \mathrm{GHz}$. The application of the previous procedure, considering the parameters of the Rogers RO3010 substrate with dielectric constant $\varepsilon_{r}=10.2$ and thickness $h=1.27 \mathrm{~mm}$, gives $C_{l s}=0.57 \mathrm{pF}, Z_{o e}=152.76 \Omega$,

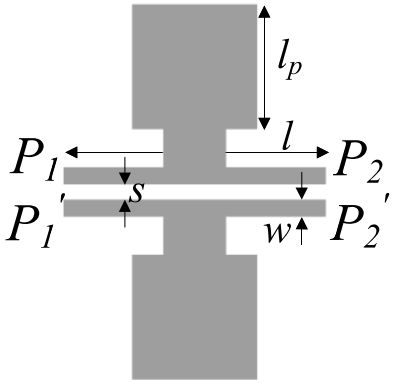

(a)

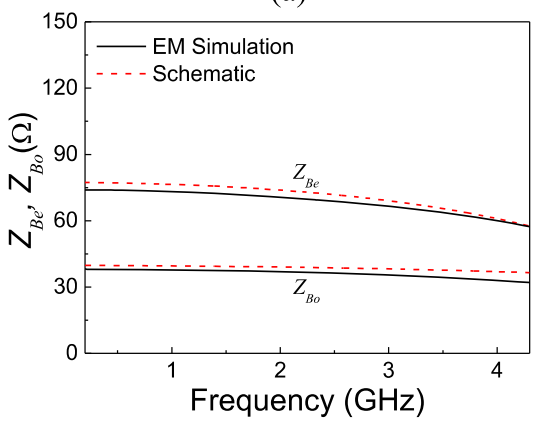

(b)

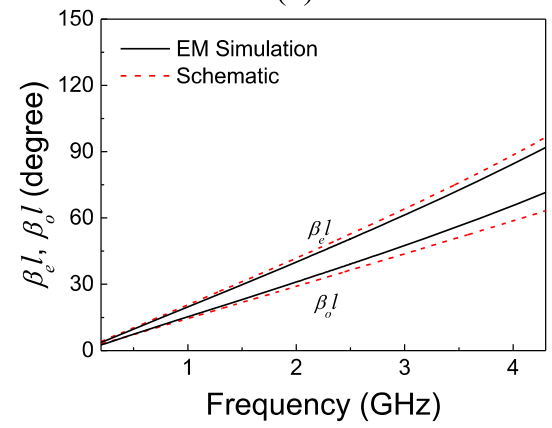

(c)

Fig. 2. (a) Layout. (b) Even- and odd-mode Bloch impedance. (c) Electrical length corresponding to the designed CLCL unit cell reported in the example. Circuit schematic is referred to Fig. 1(b). Dimensions are $l=3.962 \mathrm{~mm}$, $l_{p}=1.885 \mathrm{~mm}, w=0.252 \mathrm{~mm}$, and $s=0.238 \mathrm{~mm}$.

$Z_{o o}=58.65 \Omega, k_{e} l=26.6^{\circ}, k_{o} l=24.9^{\circ}, s w r_{o}=0.68$, $\beta_{e} l=53.2^{\circ}$, and $\beta_{o} l=36.9^{\circ}$. From these values, we have obtained the four-port S-parameters of the schematic, and from these values and well-known transformations [49], we have inferred the S-parameters for the even and odd modes. By transformation to $A B C D$ parameters, we have then obtained the Bloch impedance and electrical length for each mode [1], [48]. The results, depicted in Fig. 2, demonstrate that the target values are satisfied to a good approximation.

Once the electrical parameters of the CLCL are found, the next step is to determine the layout (unit cell). To this end, an ASM optimization algorithm is developed (it will be discussed in the next section). The seeding layout, necessary to initiate such ASM algorithm, is obtained from the value of $C_{l s}$, which gives the dimensions of the patch capacitors according to the parallel plate capacitor formula, from $Z_{o o}$ and $Z_{o e}$, which provide the width and separation of the unloaded coupled lines, and from $k_{o} l$ and $k_{e} l$, which provide their length. A commercial transmission-line calculator (such as the 

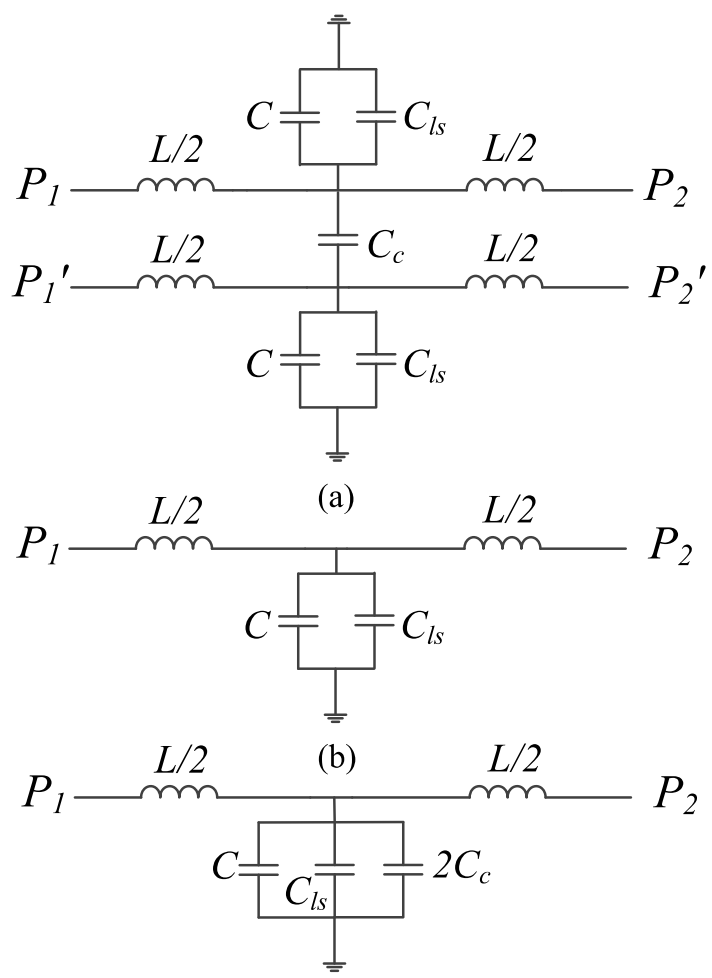

(c)

Fig. 3. (a) Lumped element circuit model (unit cell) of the CLCL. Circuit model for (b) even- and (c) odd-mode excitation.

one included in Keysight Momentum) can be used to determine the width, separation, and length of the unloaded coupled lines. As can be seen in Fig. 2, the full-wave EM simulation of the layout (unit cell) agrees very well with the results of the designed circuit schematic.

\section{B. Optimizing Spurious Suppression}

In the preceding section, it has been assumed that the number of cells per coupled line section, $N$, is not a design parameter. However, $N$ determines the onset and bandwidth of the first stopband of the CLCL (as will be shown), and hence it should not be arbitrarily chosen. $N$ must be determined according to a systematic procedure, in order to guarantee that the first and a certain number of spurious bands of the bandpass filter under design are efficiently suppressed. Such procedure, based on the approximate lumped element equivalent circuit model of the unit cell, is presented and discussed in this section. Such model, depicted in Fig. 3, assumes that the interaction between both lines is dominated by electric coupling and that the electrical length of the unit cell is small. These approximations are reasonable provided that the intention is to estimate the effects of $N$ on spurious suppression, rather than to use this model for design purposes. $L$ and $C$ are the inductance and capacitance of the lines, $C_{l s}$ is the capacitance of the square patches (as mentioned before), and $C_{c}$ is the coupling capacitance between the lines. The models for even and odd mode excitation are also included in Fig. 3.
The dispersion and Bloch impedances of the models of Fig. 1(c) and (d) and the corresponding models of Fig. 3(b) and (c) converge at low frequencies (small electrical length) and tend to diverge as the frequency approaches the cutoff frequencies of the fundamental modes (even and odd modes). Such frequencies determine the onset of the stopband for each mode, caused by the periodicity. Nevertheless, if $C_{l s} / C \gg 1$, the discrepancy between both models is very small up to the cutoff frequencies, as it has been demonstrated in reference to single-ended capacitively loaded transmission lines [1]. The previous condition $\left(C_{l s} / C \gg 1\right)$ is roughly satisfied in this paper, since one of the objectives is to achieve significant miniaturization, and this is intimately related to the ratio $C_{l s} / C$, as will be later shown.

Even though CLCLs are dispersive (as mentioned before), under the long wavelength approximation (far enough from the cutoff frequencies), it can be assumed that the even and odd mode Bloch impedances are frequency independent and given by

$$
\begin{aligned}
Z_{B e} & =\sqrt{\frac{L}{C+C_{l s}}} \\
Z_{B o} & =\sqrt{\frac{L}{C+C_{l s}+2 C_{c}}}
\end{aligned}
$$

as derived from the equivalent circuits for the even and odd modes, depicted in Fig. 3. Similarly, the electrical lengths (unit cell) for the even and odd modes can be expressed as linear functions of frequency

$$
\begin{aligned}
& \varphi_{e, \text { cell }}=\beta_{e} l=\omega \sqrt{L\left(C+C_{l s}\right)} \\
& \varphi_{o, \text { cell }}=\beta_{o} l=\omega \sqrt{L\left(C+C_{l s}+2 C_{c}\right)} .
\end{aligned}
$$

In the framework of the lumped (even and odd) models, the slow-wave factors for each fundamental mode are given by

$$
\begin{aligned}
& s w r_{e}=\frac{k_{e} l}{\beta_{e} l}=\frac{\omega \sqrt{L C}}{\omega \sqrt{L\left(C+C_{l s}\right)}}=\frac{1}{\sqrt{1+\frac{C_{l s}}{C}}} \\
& s w r_{o}=\frac{k_{o} l}{\beta_{o} l}=\frac{\omega \sqrt{L\left(C+2 C_{c}\right)}}{\omega \sqrt{L\left(C+C_{l s}+2 C_{c}\right)}}=\frac{1}{\sqrt{1+\frac{C_{l s}}{C+2 C_{c}}}} .
\end{aligned}
$$

Under low coupling level (satisfied if $C \gg 2 C_{c}$ ), the phase constants and slow-wave factors for both modes can be assumed to be roughly the same. Although it is not possible to achieve tightly coupled lines in edge coupled configurations, depending on the required filter bandwidth, the coupling level between the lines may be moderate, and therefore the above condition $\left(C \gg 2 C_{c}\right)$ is not strictly valid necessarily. Nevertheless, for the purpose of this section, we will assume that the phase constants and slow-wave factors for both modes are identical. The reason is that we do not need accurate expressions to determine the number of cells, $N$, per coupled line section.

According to the previous paragraph, (7) can be rewritten as

$$
\left.\varphi\right|_{f_{0}}=\frac{\pi}{2 N}
$$


where $\varphi=\varphi_{e}=\varphi_{o}$, in coherence with the considered approximation. In order to determine $N$, the relative position of the cutoff frequency, $f_{C}$, of the CLCLs with regard to the filter central frequency, $f_{0}$, must be taken into account. Under the considered approximation $\left(C \gg 2 C_{c}\right)$, the cutoff frequency for both modes is identical and given by [1], [18]

$$
f_{C}=\frac{1}{\pi \sqrt{L\left(C+C_{l s}\right)}} .
$$

By combining (14) and (11a), it follows that

$$
\left.\varphi\right|_{f_{0}}=2 \pi f_{0} \sqrt{L\left(C+C_{l s}\right)}=2 \frac{f_{0}}{f_{C}}
$$

and, by introducing (13) in (15), the ratio between the cutoff frequency and the filter central frequency can be expressed as

$$
\frac{f_{C}}{f_{0}}=\frac{4 N}{\pi}
$$

In coupled-line bandpass filters, the first spurious band appears at $2 f_{0}$. For the suppression of that band, it is thus necessary that $f_{C}<2 f_{0}$. Therefore, according to (16), it follows that the number of stages (unit cells) of the coupled lines, $N$, must satisfy:

$$
N<\frac{\pi}{2}
$$

giving a single stage $(N=1)$. If the number of unit cells is $N \geq 2$, then the first spurious band cannot be efficiently suppressed. However, it is not necessary that the number of unit cells of each coupled line section is the same. Expression (17) dictates that at least one of the coupled line sections must be designed with a single unit cell in order to reject the first spurious band of the filter. In practice, we have designed the filters reported in this paper by combining coupled line sections with one and two unit cells, as will be later shown.

In order to estimate the capability of the CLCL sections in rejecting multiple harmonic bands, the upper limit of the stopband must be determined. It is given by [1], [18]

$$
f_{S}=\frac{1}{2 \sqrt{L C}}
$$

where, again, it has been assumed that $f_{S}$ is identical for the even and odd modes. Note that this expression is not inferred from the lumped element equivalent circuit model of Fig. 3(b) or (c), since this model does not predict the presence of a finite stopband above $f_{C}$, but an unlimited stopband. To obtain (18), the distributed model of Fig. 1(c) or (d), under the considered low coupling level approximation, is necessary. Analysis of (1) reveals that the upper limit of the stopband occurs when $\beta_{e} l=k_{e} l=\pi$ (note that this is not in contradiction with an swr smaller than one, since the $s w r$ makes sense in the first passband of the slow-wave structure). Since

$$
k_{e} l=2 \pi f \sqrt{L C}
$$

(18) is obtained.
By dividing (18) and (14), the ratio between $f_{S}$ and $f_{C}$ is obtained as

$$
\frac{f_{S}}{f_{C}}=\frac{\pi}{2} \sqrt{1+\frac{C_{l s}}{C}}=\frac{\pi}{2} \frac{1}{s w r}
$$

with $s w r=s w r_{e}=s w r_{o}$. By introducing (16) in (20)

$$
\frac{f_{S}}{f_{0}}=\frac{2 N}{s w r} \text {. }
$$

That is, the number of spurious bands that can be rejected with $N=1$ (a value necessary to reject the first harmonic band) is determined by the slow-wave factor. Let us consider, for instance, that $s w r=1 / 2$, corresponding to $50 \%$ size reduction. With this value and (21), the stopband extends up to $f_{S}=4 f_{0}$, and hence the first $\left(2 f_{0}\right)$ and second $\left(3 f_{0}\right)$ spurious bands are expected to be efficiently suppressed.

Let us now consider that $N=2$. In this case, (16) and (21) give $f_{C}=2.55 f_{0}$ and $f_{S}=8 f_{0}$, respectively. Hence, we expect that coupled line sections with two capacitive stages are able to reject from the second $\left(3 f_{0}\right)$ up to the sixth $\left(7 f_{0}\right)$ spurious band. Note that these results indicate that by combining CLCL sections with single and two capacitive unit cells in the considered filter, a very wide stopband (provided the slow-wave ratio is set to $s w r_{e}=1 / 2$ ) should result. This is the considered situation in the examples reported in Section IV.

\section{ASM OPTIMIZATION TOOL}

ASM is an optimization tool that has been applied in many different scenarios, in particular to the synthesis of planar microwave circuits that can be described by lumped element equivalent circuits, or by a combination of lumped and distributed (transmission-line sections or stubs) elements [50]-[54]. In ASM optimization, two different simulation spaces are considered: 1) the optimization space, $\mathbf{X}_{\mathbf{c}}$, where the variables are linked to a coarse model, which is simple and computationally efficient, although not accurate and 2) the validation space, $\mathbf{X}_{\mathbf{f}}$, where the variables are linked to a fine model, typically more complex and CPU intensive, but substantially more precise. In each space, a vector containing the different model parameters is defined. Such vectors are denoted by $\mathbf{x}_{\mathbf{f}}$ and $\mathbf{x}_{\mathbf{c}}$ for the fine and coarse model spaces, respectively, and their corresponding responses are designated as $\mathbf{R}_{\mathbf{f}}\left(\mathbf{x}_{\mathbf{f}}\right)$ and $\mathbf{R}_{\mathbf{c}}\left(\mathbf{x}_{\mathbf{c}}\right)$.

In the optimization of planar circuits, the variables of the optimization space are typically a set of lumped elements (inductors and/or capacitors) [52], a set of electrical parameters (e.g., electrical length or characteristic impedance of a transmission line), or a combination of the previous elements/parameters [50], [53], [54]. In this space, the response is inferred from the electrical simulation of the lumped element circuit or circuit schematic. The variables of the validation space are a set of geometrical parameters that define the circuit layout, and the response in this space is obtained from the electromagnetic simulation of that layout. If the number of geometrical parameters necessary to completely define the geometry is too high, some of them are fixed to certain (reasonable) values [53], [54]. Moreover, substrate parameters are 
usually fixed, and hence they are not optimization variables. In ASM, it is very convenient to deal with the same number of parameters in both spaces since this eases the implementation of the ASM algorithm.

In ASM, the target solution is determined through an iterative quasi-Newton type algorithm, and a parameter transformation mapping the fine model parameter space to the coarse model parameter space is needed. In planar circuits, such parameter transformation is simply a parameter extractor, providing the elements of the coarse model from the response of the fine model (e.g., the element values of the circuit model, inferred from the electromagnetic simulation of the circuit layout by applying specific parameter extraction techniques). Thus, the goal in ASM is to minimize the following error function:

$$
\mathbf{f}\left(\mathbf{x}_{\mathbf{f}}\right)=\mathbf{P}\left(\mathbf{x}_{\mathbf{f}}\right)-\mathbf{x}_{\mathbf{c}}^{*}
$$

where $\mathbf{x}_{\mathbf{c}}^{*}$ is the coarse model solution that gives the target response, $\mathbf{R}_{\mathbf{c}}\left(\mathbf{x}_{\mathbf{c}}^{*}\right)$, and $\mathbf{P}\left(\mathbf{x}_{\mathbf{f}}\right)$ provides the coarse model parameters from the fine model parameters by means of a parameter extraction procedure, as mentioned above (examples of parameter extraction in transmission lines loaded with electrically small resonators can be found in [55] and [56]).

Let us assume that $\mathbf{x}_{\mathbf{f}}^{(j)}$ is the $j$ th approximation to the solution in the validation space and $\mathbf{f}^{(j)}$ is the error function corresponding to $\mathbf{f}\left(\mathbf{x}_{\mathbf{f}}^{(j)}\right)$. The next vector of the iterative process $\mathbf{x}_{\mathbf{f}}^{(j+1)}$ is obtained by a quasi-Newton iteration according to

$$
\mathbf{x}_{\mathbf{f}}^{(j+1)}=\mathbf{x}_{\mathbf{f}}^{(j)}+\mathbf{h}^{(j)}
$$

where $\mathbf{h}^{(j)}$ is given by

$$
\mathbf{h}^{(j)}=-\left(\mathbf{B}^{(j)}\right)^{-1} \mathbf{f}^{(j)}
$$

and $\mathbf{B}^{(j)}$ is an approach to the Jacobian matrix, which is updated according to the Broyden formula [42]

$$
\mathbf{B}^{(j+1)}=\mathbf{B}^{(j)}+\frac{\mathbf{f}^{(j+1)} \mathbf{h}^{(j) T}}{\mathbf{h}^{(j) T} \mathbf{h}^{(j)}} .
$$

In (25), $\mathbf{f}^{(j+1)}$ is obtained by evaluating (22), and the superindex $T$ stands for transpose.

In complex circuits, such as filters, dealing with all the variables of both spaces simultaneously may be too cumbersome. However, if the structure to be designed consists of several uncoupled stages or sections, it is possible to optimize each section separately, then cascade or group them, and postoptimize if necessary. Moreover, within each circuit section, if there is small correlation between certain elements of both spaces, it is potentially possible to divide the algorithm in different ASM subprocesses. This approach reduces complexity and computational time, and has been successfully applied, e.g., to the design of bandpass filters based on stepped impedance resonators [53], [54].

In this paper, the considered filters are implemented by cascading CLCL sections, and we assume that intersection coupling is negligible. Therefore, each coupled line section is synthesized independently. In addition, the dimensions of the capacitive patches are optimized independently, using a

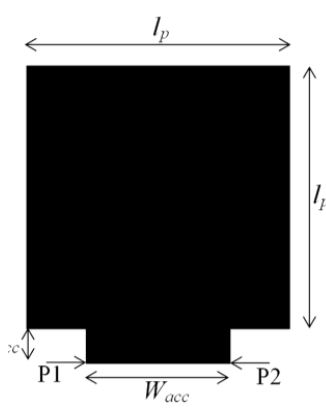

Fig. 4. Topology and relevant dimensions of the capacitive patch.

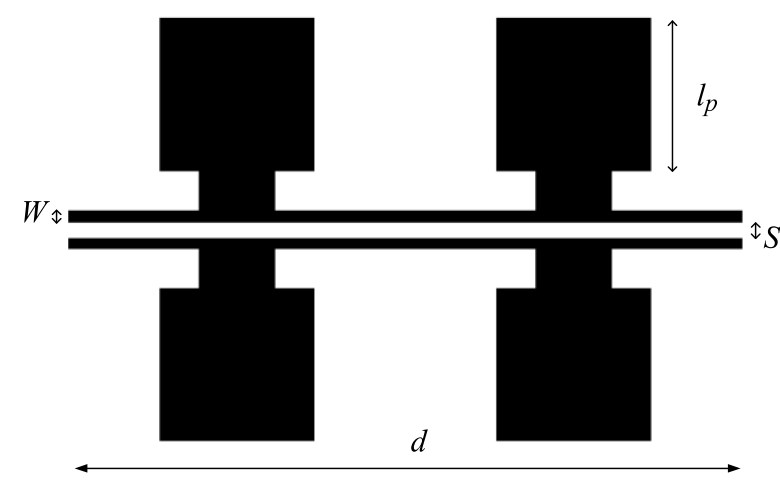

Fig. 5. Topology and relevant dimensions of the patch-loaded coupled-line section, where two unit cells have been considered.

one-variable (for each space) ASM subprocess developed in [50]. This means that we first determine patch dimensions, and such dimensions are not optimization variables in the ASM algorithm specifically developed to obtain the whole coupled line section layout. For convenience, let us designate the first one-variable ASM subalgorithm as patch-ASM, and the second one, involving the whole CLCL section, as CLCL-ASM.

In brief (see further details in [50]), the variables of the fine and coarse models in the patch-ASM are the side length $\left(l_{p}\right)$ of the capacitive patch and the capacitance $C_{l s}$, respectively. The geometry is depicted in Fig. 4, where a square-shaped patch connected to the host line through a wide and short access strip (to avoid any inductive effect) is considered. The distance between the host line and the patch (length of the access strip, $\left.l_{\text {acc }}\right)$ is set to a fixed value $(0.6 \mathrm{~mm})$, whereas its width $\left(W_{\mathrm{acc}}\right)$ is set to a fraction of the patch dimension $l_{p}$ (specifically, it has been found that $50 \%$ of the patch dimension is a good choice). The first value of $l_{p}$ is inferred from the target value of $C_{l s}$ (derived from the procedure explained in Section II), through the parallel plate capacitor formula. Then the capacitance of this patch is inferred from the electromagnetic simulation by considering the ports indicated in Fig. 4, specifically by inferring the admittance of the shunt load. Once the capacitance is obtained, it is compared with the target value, and the iterative ASM process is started-using the expression in (23) - unless the error is smaller than a certain predefined value.

For the CLCL-ASM algorithm, the variables in the validation space are the width, $W$, separation, $S$, and length, $d$, of the unloaded coupled lines (Fig. 5). Note that the length $d$ must satisfy $d=N \cdot l$. In principle, the natural variables 
in the optimization space should be the even and odd mode Bloch impedances $\left(Z_{B e}\right.$ and $\left.Z_{B o}\right)$ of the CLCLs, and the average electrical length of the two modes at $f_{0}$, i.e., $\varphi_{a v}=$ $N\left(\varphi_{e}+\varphi_{o}\right) / 2$ [see (7)]. The reason is that the target values of these impedances $\left(Z_{B e}^{*}\right.$ and $\left.Z_{B o}^{*}\right)$ for each coupled line section are directly dictated by filter specifications, and the average electrical length of the loaded coupled lines is usually forced to be $\varphi_{a v}^{*}=\pi / 2$. Nevertheless, we have actually considered different optimization variables, to be specified next.

We terminate in open circuits two crossed ports of the patchloaded coupled lines, so that a two-port circuit results. Assuming that the electrical lengths of both modes are identical, i.e., $\varphi=N \varphi_{e}=N \varphi_{o}$, the image impedance of this circuit is related to $Z_{B e}, Z_{B o}$, and $\varphi$ by [48]

$$
Z_{I}=\frac{1}{2} \sqrt{\left(Z_{B e}-Z_{B o}\right)^{2} \csc ^{2} \varphi-\left(Z_{B e}+Z_{B o}\right)^{2} \cot ^{2} \varphi}
$$

and it takes the maximum value when $\varphi=\pi / 2$ as

$$
Z_{I, \max }=\frac{Z_{B e}-Z_{B o}}{2} \text {. }
$$

Moreover, the real part of the image impedance around $\varphi=\pi / 2$ is delimited to a band with cutoffs, $\varphi_{1}$ and $\varphi_{2}$, given by

$$
\cos \varphi_{1}=-\cos \varphi_{2}=\frac{Z_{B e}-Z_{B o}}{Z_{B e}+Z_{B o}} .
$$

Note that, from the target values of $Z_{B e}^{*}$ and $Z_{B o}^{*}$, we can define target values for the maximum image impedance, $Z_{I, \max }^{*}$, and for the phase of the lower cutoff frequency, $\varphi_{1}^{*}$. The image impedance can be inferred from the simulated $S$-parameters of the constructed two-port structure by conversion to $A B C D$ parameters

$$
Z_{I}=\frac{B}{\sqrt{A^{2}-1}}
$$

and from it, we can identify $Z_{I, \max }$ and $\varphi_{1}$. Using (27) and (28), $Z_{B e}$ and $Z_{B o}$ can be isolated and thus extracted. However, $Z_{I, \max }$ and $\varphi_{1}$ (rather than $Z_{B e}$ and $Z_{B o}$ ) are the considered optimization variables in the coarse space.

The phase $\varphi$ of the pair of patch-loaded coupled lines is related to the phase of the two-port structure, $\psi$, by [43]

$$
\cos \psi=\frac{Z_{B e}+Z_{B o}}{Z_{B e}-Z_{B o}} \cos \varphi .
$$

Moreover

$$
\cos \psi=\frac{A+D}{2}=A
$$

since $A=D$ in our case. Hence, from the S-parameters and transformation to $A B C D$ parameters, the phase of the two port structure, $\psi$, can be inferred, and from it, we can obtain $\varphi$ using (30). Note that, according to (30), $\psi=\pi / 2$ gives $\varphi=\pi / 2$ and vice versa. Hence, we can directly use $\psi$ for optimization purposes, and consider the frequency where $\psi=\pi / 2$, i.e., $f_{\pi / 2}$, as the third variable of the coarse space. Obviously, the target is $f_{\pi / 2}^{*}=f_{0}$.

In the previous procedure, the involved formulation is strictly valid if the phases of the two modes are identical, which is not the case, as mentioned before. However, the values of $Z_{B e}$ and $Z_{B o}$ that result by considering the CLCLs as a two-port [i.e., by isolation from (27) and 28)] are in good approximation to those inferred from the procedure explained in Section II-A, and (30) represents a phase condition for the electrical length of the two modes as reasonable as (7). Note, however, that forcing $\psi=\pi / 2$ does not mean that the average values of the electrical lengths are strictly $\pi / 2$ (but roughly that value). Nevertheless, it has been found that with this procedure, the coupled line filter sections, once cascaded, provide a filter response very close to the target.

Once the three variables of the optimization and validation spaces have been determined, the CLCL-ASM algorithm is implemented. As mentioned before, the first layout is generated from the values of $Z_{o e}, Z_{o o}$ and $N k_{e} l, N k_{o} l$ derived from the procedure indicated in Section II and a transmission line calculator. Since the dimensions of the patch capacitors are inferred from an independent ASM subprocess (patch-ASM), such dimensions are those that are considered in the first and successive layouts of the whole CLCL section. From the seeding layout, the parameters of the coarse model $\left(Z_{I, \max }, \varphi_{1}\right.$, and $\left.f_{\pi / 2}\right)$ are extracted and subsequently compared with the target values in order to infer the first error function. Next, the new layout of the iterative process is inferred from (23), with $\mathbf{h}$ given by (24) and the initial Broyden matrix calculated as

$$
\mathbf{B}=\left(\begin{array}{ccc}
\frac{\delta Z_{I, \max }}{\delta W} & \frac{\delta Z_{I, \max }}{\delta S} & \frac{\delta Z_{I, \max }}{\delta d} \\
\frac{\delta \varphi_{1}}{\delta W} & \frac{\delta \varphi_{1}}{\delta S} & \frac{\delta \varphi_{1}}{\delta d} \\
\frac{\delta f_{\pi / 2}}{\delta W} & \frac{\delta f_{\pi / 2}}{\delta S} & \frac{\delta f_{\pi / 2}}{\delta d}
\end{array}\right) .
$$

The process is then iterated until convergence is obtained. In this paper, the algorithm ends when the following norm:

$$
\left\|f_{\text {norm }}\right\|=\sqrt{\left(\frac{f_{\pi / 2}}{f_{\pi / 2}^{*}}-1\right)^{2}+\left(\frac{\varphi_{1}}{\varphi_{1}^{*}}-1\right)^{2}+\left(\frac{Z_{I, \max }}{Z_{I, \max }^{*}}-1\right)^{2}}
$$

is smaller than $0.5 \%$.

\section{DESIGN EXAMPLES}

\section{A. Third-Order Filter}

Using the previously reported ASM algorithm, we have generated the layout of a third-order filter consisting of four cascaded CLCL sections, as a first illustrative example. A Chebyshev response was considered with central frequency $f_{0}=1.0 \mathrm{GHz}$, fractional bandwidth $\mathrm{FBW}=8 \%$, and ripple level of $L_{A r}=0.1 \mathrm{~dB}$. With these specifications, the impedances of the coupled line sections were found to be $Z_{B e}=73.54 \Omega$ and $Z_{B o}=38.64 \Omega$ for coupled line sections 1 and 4 , and $Z_{B e}=56.44 \Omega$ and $Z_{B o}=44.89 \Omega$ for coupled line sections 2 and 3 . The layout of the conventional filter, inferred from a simplified version of the proposed ASM (provided that the patch capacitors are not present), is depicted in Fig. 6(a). The considered substrate is the Rogers RO3010, with thickness $h=1.27 \mathrm{~mm}$ and dielectric constant $\varepsilon_{\mathrm{r}}=10.2$, and electromagnetic simulations were carried out by means of 


\section{$114.11 \mathrm{~mm}$}

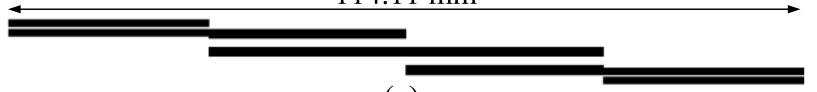

(a)

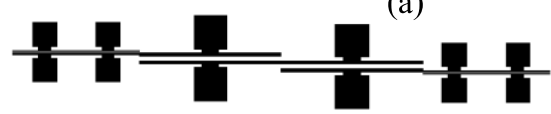

$77.01 \mathrm{~mm}$ (b)

Fig. 6. Layouts of the designed coupled-line bandpass filters. (a) Conventional. (b) Capacitively loaded. Both layouts are drawn to the same scale.

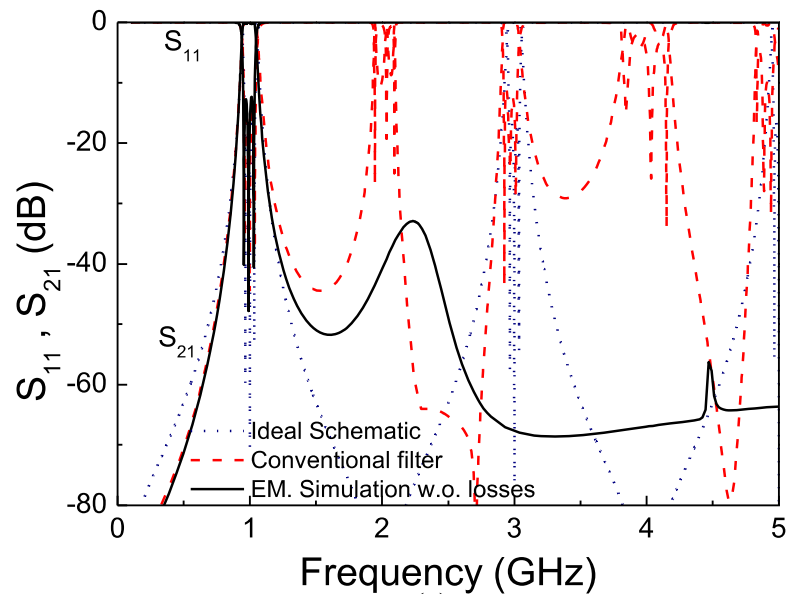

(a)

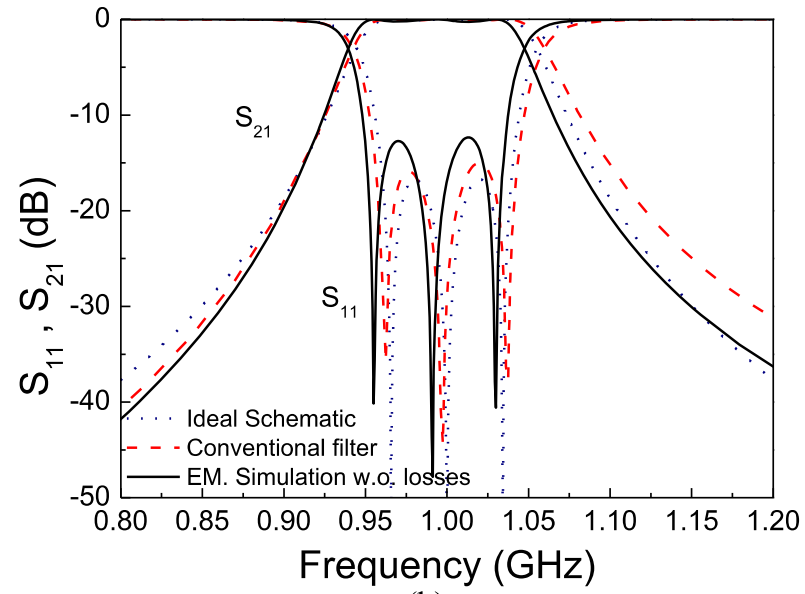

(b)

Fig. 7. Lossless electromagnetic response of the conventional and CLCL bandpass filters of Fig. 6. (a) Wideband response. (b) Zoomed-in view of the passband.

the Keysight Momentum commercial simulator. The frequency response is depicted in Fig. 7, where the presence of spurious bands, at $2 f_{0}$ and at higher harmonic frequencies, can be appreciated. Such filter has been designed for comparison purposes. Moreover, a very good agreement in terms of central frequency, bandwidth, and the ripple level can be observed.

For the design of the CLCL bandpass filter, a single unit cell for filter sections 2 and 3 has been considered, whereas for sections 1 and 4, the coupled lines are loaded with two pairs of patches. By this procedure, we ensure a wide stopband, as discussed in Section II. The slow-wave ratio has been
TABLE I

ElEMENTS OF THE SCHEMATIC OF FIG. 1 USED TO DETERMINE THE SEEDING LAYOUT of THE ASM

\begin{tabular}{c|c|c|c|c|c}
\hline Stage & $\boldsymbol{Z}_{\boldsymbol{o e}}(\Omega)$ & $\boldsymbol{Z}_{\boldsymbol{o o}}(\Omega)$ & $\boldsymbol{C}_{\boldsymbol{l s}}(\mathrm{pF})$ & $\boldsymbol{k}_{\boldsymbol{e}} \boldsymbol{l}$ & $\boldsymbol{k}_{\boldsymbol{o}} \boldsymbol{l}$ \\
\hline 1,4 & 154.84 & 57.65 & 1.293 & $25.23^{\circ}$ & $23.68^{\circ}$ \\
2,3 & 145.48 & 79.59 & 2.349 & $50.66^{\circ}$ & $47.36^{\circ}$ \\
\hline
\end{tabular}

TABLE II

ELEMENTS OF THE FINE MODEL FOR THE INITIAL LAyOUT

\begin{tabular}{c|c|c|c|c}
\hline Stage & $\boldsymbol{W}(\mathrm{mm})$ & $\boldsymbol{S}(\mathrm{mm})$ & $\boldsymbol{d}(\mathrm{mm})$ & $\boldsymbol{l}_{\boldsymbol{p}}$ \\
\hline 1,4 & 0.1220 & 0.1950 & 16.648 & 4.264 \\
2,3 & 0.0980 & 0.4840 & 16.619 & 5.747 \\
\hline
\end{tabular}

TABLE III

Elements of the Fine Model After Convergence

\begin{tabular}{c|c|c|c|c}
\hline Stage & $\boldsymbol{W}(\mathrm{mm})$ & $\boldsymbol{S}(\mathrm{mm})$ & $\boldsymbol{d}(\mathrm{mm})$ & $\boldsymbol{l}_{\boldsymbol{p}}$ \\
\hline 1,4 & 0.1703 & 0.1950 & 18.193 & 3.432 \\
2,3 & 0.3729 & 0.7866 & 20.312 & 4.754 \\
\hline
\end{tabular}

TABLE IV

Elements of the Coarse Model After Convergence

\begin{tabular}{c|c|c|c|c}
\hline Stage & $\boldsymbol{Z}_{I, \max }(\Omega)$ & $\boldsymbol{\varphi}_{1}$ & $\boldsymbol{f}_{\pi / 2}(\mathrm{GHz})$ & $\boldsymbol{C}_{\boldsymbol{l}}(\mathrm{pF})$ \\
\hline 1,4 & 17.466 & $72.06^{\circ}$ & 1.002 & 1.293 \\
2,3 & 5.755 & $83.52^{\circ}$ & 0.998 & 2.359 \\
\hline
\end{tabular}

considered to be $s w r_{e}=0.5$. Using (1)-(9), the value of the patch capacitances and the electrical parameters of the coupled line sections can be found (see Table I).

Moreover, the resulting slow-wave ratio for the odd mode and electrical lengths of the CLCL sections are $s w r_{o}=0.682$, $\beta_{e} l=50.47^{\circ}$, and $\beta_{o} l=34.72^{\circ}$, respectively, for sections 1 and 4 , and for sections 2 and 3, they are $s w r_{o}=0.625, \beta_{e} l=101.32^{\circ}$, and $\beta_{o} l=75.78^{\circ}$. With these values, the parameters of the fine model inferred from a transmission line calculator (except $l_{p}$, which has been obtained by means of the parallel plate capacitor formula) have been found to be the ones indicated in Table II. Note that the average value of $\beta_{e} l$ and $\beta_{o} l$ is not $\pi / 4$ (sections 1 and 4) or $\pi / 2$ (sections 2 and 3 ). The reason is that, rather than (7), we have actually forced $\psi=\pi / 2$, since this provides a seeding layout closer to the final one for each filter stage. By forcing $\psi=\pi / 2$, the average values of the electrical lengths of the coupled lines are not necessarily $\pi / 2$, as mentioned before.

Once the seeding layout has been inferred, application of the patch-ASM has provided a side length of $l_{p}=3.432 \mathrm{~mm}$ for the capacitors of sections 1 and 4 , and $l_{p}=4.754 \mathrm{~mm}$ for the capacitors of sections 2 and 3. Application of the CLCL-ASM has provided the final filter layout after 12 and 5 iterations for the synthesis of the coupled line sections with a single and two unit cells, respectively. The variables in each space after convergence are indicated in Tables III and IV.

Fig. 8 shows the image impedance and the phase $\psi$ at several iterations for the coupled line sections with one and two unit cells, where it can be appreciated that the curves progressively evolve so that the variables of the coarse model tend to the target values, also indicated. The final layout of 

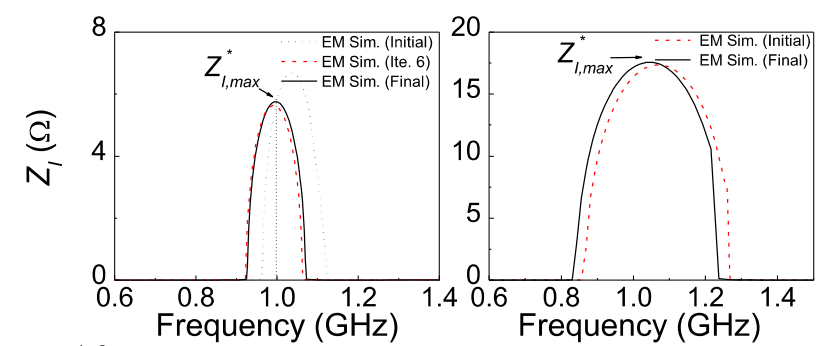

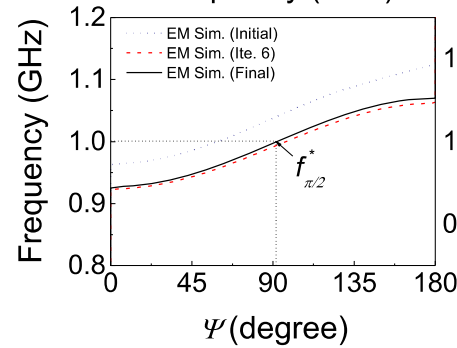

(a)

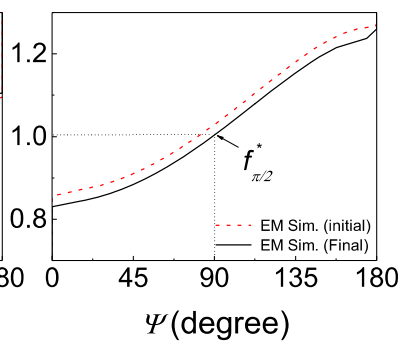

(b)
Fig. 8. Image impedance and phase $\psi$ of the coupled-line sections at various iterations. (a) Single unit cell sections. (b) Two unit cell sections.

the filter generated by simply cascading the different coupled line sections is depicted in Fig. 6(b). Substantial size reduction compared with the conventional filter is achieved by virtue of the considered slow-wave factor. The electromagnetic simulation of this filter is depicted in Fig. 7. It can be seen that the response in the region of interest is very similar to the one of the ordinary filter. However, the spurious bands are efficiently suppressed up to very high frequencies. It is also important to mention that no further optimization has been required to achieve such response.

The designed device has been fabricated by means of an LPKF H-10O drilling machine. The fabricated device and the measured frequency response, inferred by means of the Agilent PNA N5221A vector network analyzer, are depicted in Fig. 9, where the electromagnetic simulation with losses is also included for comparison. A reasonable agreement between both responses can be observed, and spurious band rejection is better than $30 \mathrm{~dB}$ up to at least $5 f_{0}$.

In order to further justify the potential of the proposed approach to reject spurious bands, and particularly the effects of the CLCL sections with $N=2$ (two capacitive stages), we have considered the CLCL sections 1 and 4 of the filter in Fig. 6(b). Specifically, we have carried out the simulation of the schematic (i.e., with ideal capacitors and coupled lines) corresponding to the even mode, by means of Keysight ADS. Fig. 10 shows the dependence of the characteristic even mode impedance, $Z_{B e}$, of the CLCL section with frequency. It can be appreciated that the value at $1 \mathrm{GHz}$ (filter central frequency) is close to the design value indicated before $\left(Z_{B e}=73.54 \Omega\right)$, but the important aspect in terms of spurious rejection is that a stopband between 2.54 and $7.14 \mathrm{GHz}$ appears. These values are in reasonable agreement with the predictions of Section II-B (see the last paragraph). We have also simulated the $S_{21}$ response of this CLCL section seen as two-port, and the results, shown in Fig. 10(b), confirm the capability of spurious attenuation.

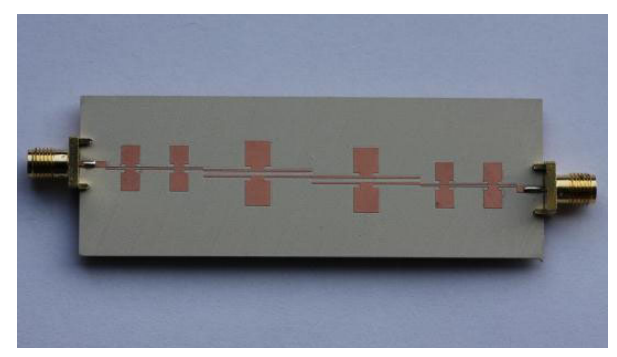

(a)

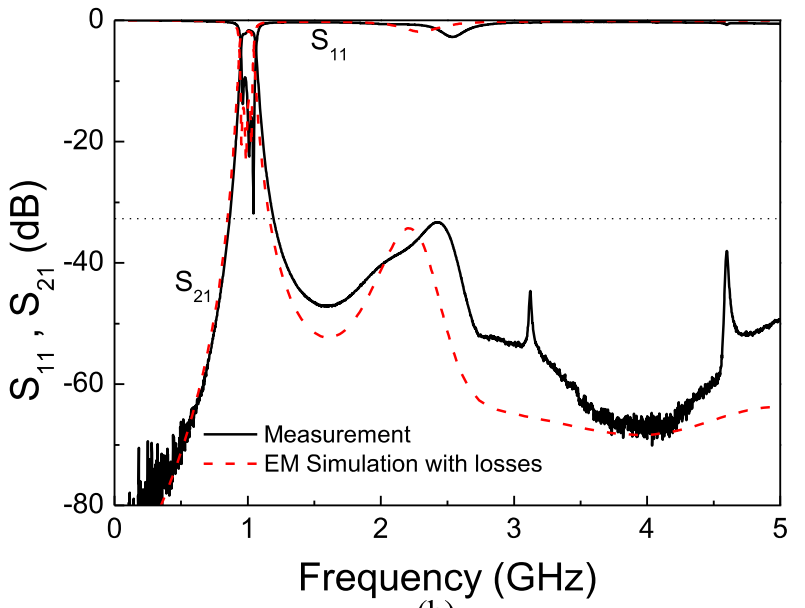

(b)

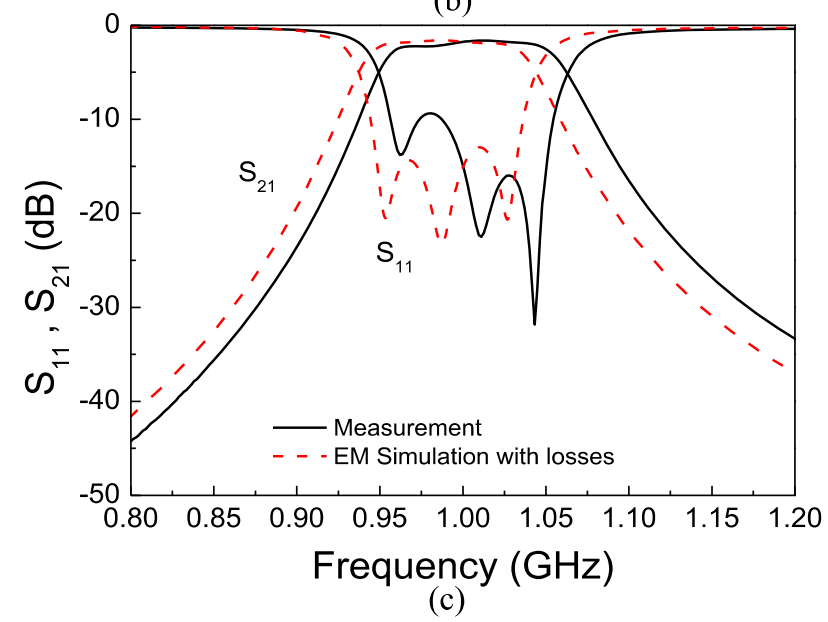

Fig. 9. (a) Fabricated third-order CLCL bandpass filter. (b) Frequency response. (c) Zoomed-in view of the passband.

\section{B. Fifth-Order Filter}

To demonstrate the potential of the proposed approach based on ASM optimization, a second illustrative example was considered. Specifically, a fifth-order Chebyshev bandpass filter consisting of six cascaded CLCL sections has been designed with the following specifications: $f_{0}=1.8 \mathrm{GHz}$, $\mathrm{FBW}=8 \%$, and ripple level of $L_{A r}=0.1 \mathrm{~dB}$. Accordingly, the impedances of the coupled line sections were found to be $Z_{B e}=72.03 \Omega$ and $Z_{B o}=38.93 \Omega$ for coupled line sections 1 and $6, Z_{B e}=55.51 \Omega$ and $Z_{B o}=45.49 \Omega$ for coupled line sections 2 and 5 , and $Z_{B e}=54.11 \Omega$ and $Z_{B o}=$ $46.47 \Omega$ for coupled line sections 3 and 4 . Once the final layout is generated (by considering a Rogers RO3010 substrate, with thickness $h=1.27 \mathrm{~mm}$ and dielectric constant $\varepsilon_{\mathrm{r}}=10.2$ ), the 


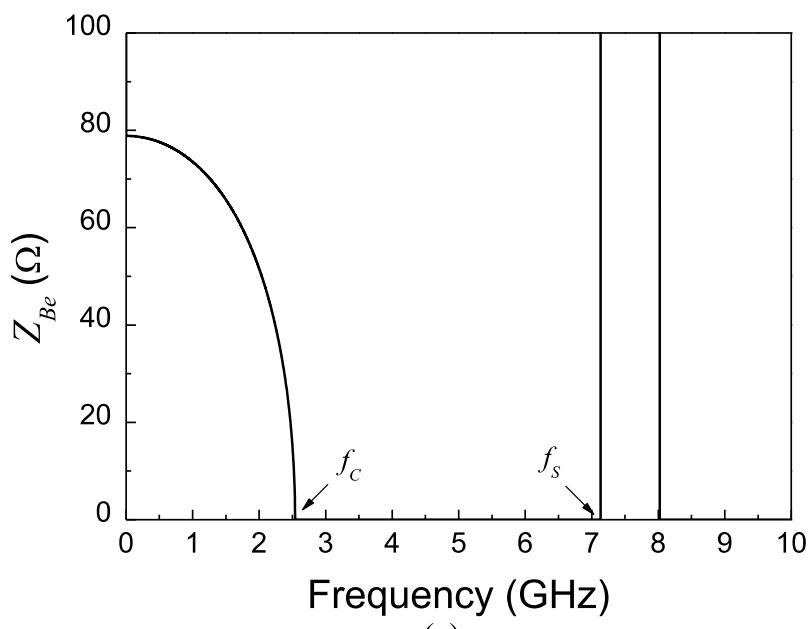

(a)

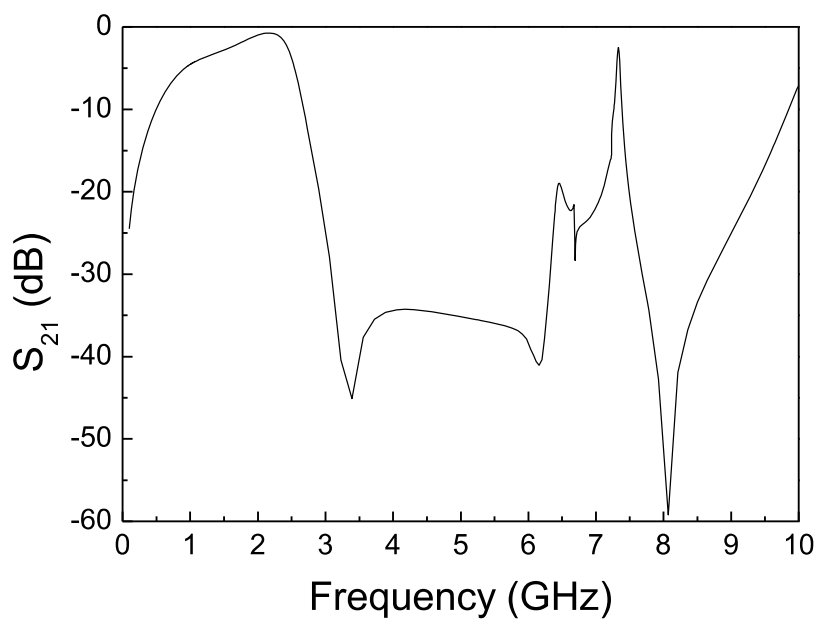

(b)

Fig. 10. (a) Even-mode characteristic impedance of the CLCL sections 1 and 4 of the filter in Fig. 6(b). (b) Response of $S_{21}$ of the same structure seen as two-port.

comparison between the lossless electromagnetic frequency response of the obtained CLCL filter and the conventional filter is shown in Fig. 11. It can be observed that there is a very good agreement between the responses in terms of central frequency, bandwidth, and the ripple level, but the capability to suppress spurious band in the CLCL-based filter is apparent.

For the design of the fifth-order CLCL bandpass filter, a single unit cell for filter sections $2-5$ has been considered, whereas for sections 1 and 6 , the coupled lines are loaded with two pairs of patches in order to suppress efficiently the spurious bands, as discussed in Section II. In this example, the slow-wave ratio for the even mode of each coupled line section is also set to $s w r_{e}=0.5$. With this value, the patch capacitances and the electrical parameters of the unloaded coupled line sections are indicated in Table V.

Furthermore, the resulting slow-wave ratio for the odd mode, $s w r_{o}$, and electrical lengths of the CLCL sections $\left(\beta_{e} l\right.$ and $\left.\beta_{o} l\right)$ are as follows. For sections 1 and 6: $s w r_{o}=$ $0.674 \beta_{e} l=42.37^{\circ}$, and $\beta_{o} l=35.02^{\circ}$, for sections 2 and 5: $s w r_{o}=0.610, \beta_{e} l=100.47^{\circ}$, and $\beta_{o} l=79.93^{\circ}$, and for sections 3 and 4 : $s w r_{o}=0.587, \beta_{e} l=99.24^{\circ}$, and

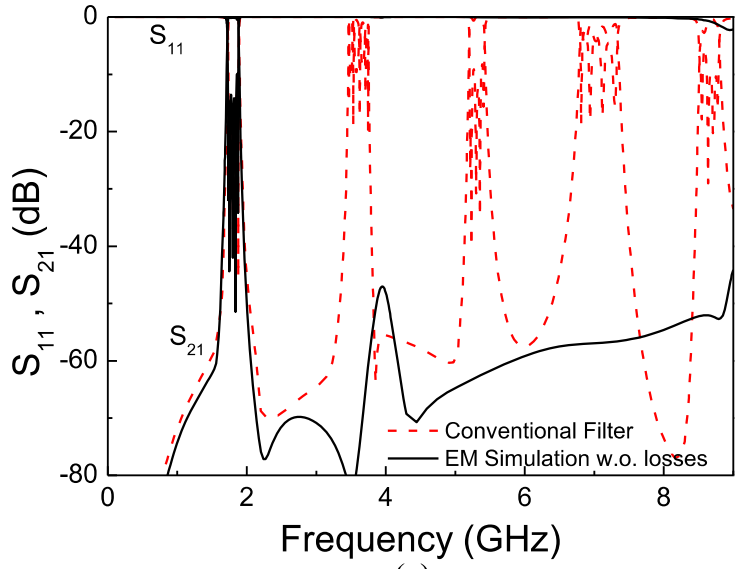

(a)

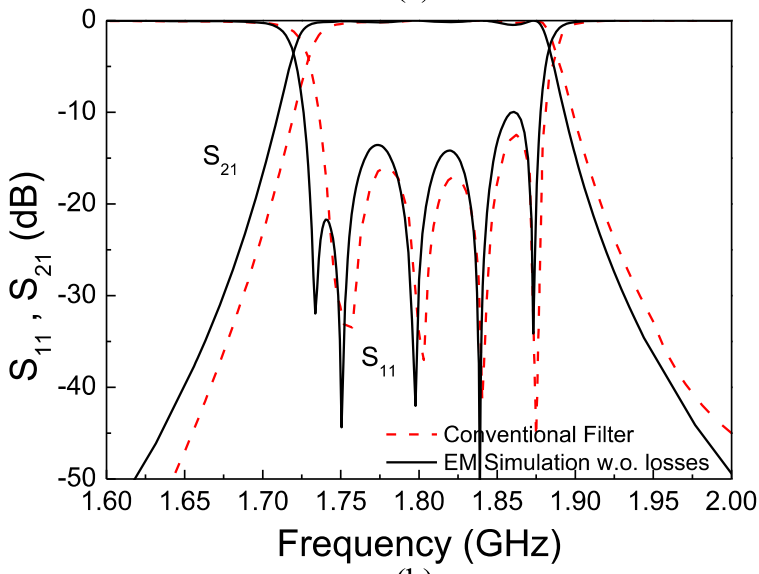

(b)

Fig. 11. Frequency responses of the conventional and CLCL fifth-order bandpass filters. (a) Wideband response. (b) Zoomed-in view of the passband.

TABLE V

Elements of the Schematic of Fig. 2 Used to Determine THE SEEDING LAYOUT OF THE ASM

\begin{tabular}{c|c|c|c|c|c}
\hline Stage & $\boldsymbol{Z}_{\boldsymbol{o} \boldsymbol{e}}(\Omega)$ & $\boldsymbol{Z}_{\boldsymbol{o} \boldsymbol{o}}(\Omega)$ & $\boldsymbol{C}_{\boldsymbol{l}}(\mathrm{pF})$ & $\boldsymbol{k}_{\boldsymbol{e}} \boldsymbol{l}$ & $\boldsymbol{k}_{\boldsymbol{o}} \boldsymbol{l}$ \\
\hline 1,6 & 151.63 & 58.79 & 0.732 & $25.18^{\mathrm{o}}$ & $23.61^{\circ}$ \\
2,5 & 142.30 & 83.24 & 1.328 & $50.24^{\circ}$ & $46.92^{\circ}$ \\
3,4 & 137.64 & 89.48 & 1.364 & $49.62^{\circ}$ & $46.323^{\circ}$ \\
\hline
\end{tabular}

$\beta_{o} l=78.92^{\circ}$. With these values, the parameters of the fine model have been found to be the ones indicated in Table VI.

The variables in each space after convergence are indicated in Tables VII and VIII. A prototype has been fabricated [see Fig. 12(a)] and measured using the aforementioned procedure. A reasonable agreement between the electromagnetic simulation (with losses) and measured frequency response of the proposed fifth-order CLCL filter can be observed in Fig. 12(b). Spurious band rejection is better than $45 \mathrm{~dB}$ up to at least $4 f_{0}$. As an added value, the final device size has been reduced by a factor of $70 \%$ compared with the conventional counterpart.

\section{Comparison With Other Approaches}

As mentioned in Section I, coupled-line bandpass filters with spurious suppression based on periodic or quasi-periodic structures with transverse width modulation were reported in [4] and [14], respectively. These filters provide very efficient spurious cancellation, specially the filter reported in [14], 
TABLE VI

ELEMENTS OF THE FINE MODEL FOR THE INITIAL LAYOUT

\begin{tabular}{c|l|l|l|c}
\hline Stage & $\boldsymbol{W}(\mathrm{mm})$ & $\boldsymbol{S}(\mathrm{mm})$ & $\boldsymbol{d}(\mathrm{mm})$ & $\boldsymbol{l}_{\boldsymbol{p}}$ \\
\hline 1,6 & 0.1270 & 0.2190 & 9.2180 & 3.208 \\
2,5 & 0.0965 & 0.5723 & 9.1430 & 4.321 \\
3,4 & 0.0930 & 0.7480 & 9.0140 & 4.380
\end{tabular}

TABLE VII

Elements of the Fine Model After Convergence

\begin{tabular}{c|c|c|c|c}
\hline Stage & $\boldsymbol{W}(\mathrm{mm})$ & $\boldsymbol{S}(\mathrm{mm})$ & $\boldsymbol{d}(\mathrm{mm})$ & $\boldsymbol{l}_{\boldsymbol{p}}$ \\
\hline 1,6 & 0.2053 & 0.2319 & 10.213 & 2.360 \\
2,5 & 0.4010 & 1.0296 & 11.349 & 3.276 \\
3,4 & 0.4270 & 1.3431 & 11.292 & 3.326 \\
\hline
\end{tabular}

TABLE VIII

Elements of the CoArse Model After Convergence

\begin{tabular}{c|c|c|c|c}
\hline Stage & $\boldsymbol{Z}_{I, \max }(\Omega)$ & $\boldsymbol{\varphi}_{1}$ & $\boldsymbol{f}_{\pi / 2}(\mathrm{GHz})$ & $\boldsymbol{C}_{\boldsymbol{l s}}(\mathrm{pF})$ \\
\hline 1,6 & 16.5187 & $72.97^{\circ}$ & 1.8045 & 0.7321 \\
2,5 & 4.9959 & $84.44^{\circ}$ & 1.7955 & 1.3283 \\
3,4 & 3.8043 & $85.52^{\circ}$ & 1.800 & 1.3644 \\
\hline
\end{tabular}

TABLE IX

COMPARISON OF VARIOUS BANDPASS FILTERS WITH SPURIOUS SUPPRESSION

\begin{tabular}{|c|c|c|c|c|c|}
\hline Ref. & $\begin{array}{l}\text { Filter } \\
\text { order }\end{array}$ & $\begin{array}{c}\text { Length } \\
\text { reduction }^{(b)}\end{array}$ & $\begin{array}{l}\text { Lumped } \\
\text { elements }\end{array}$ & $\begin{array}{c}\text { Spurious } \\
\text { suppression }\end{array}$ & $\begin{array}{l}\text { Rejection } \\
\text { level (dB) }\end{array}$ \\
\hline [14] & 7 & No & No & $1^{\text {st }}-5^{\text {th }}$ & 30 \\
\hline [57] & 3 & No & No & $1^{\mathrm{st}}$ & 40 \\
\hline [58] & 3 & $25 \%$ & No & $1^{\text {st }}-2^{\text {nd }}$ & 50 \\
\hline [59] & 3 & -- & Yes & $1^{\text {st }}-2^{\text {nd }}$ & 50 \\
\hline$[61]$ & 3 & $35 \%$ & Yes & $1^{\text {st }}-6^{\text {th }}$ & 40 \\
\hline$[62]^{(a)}$ & 3 & $90 \%$ & Yes & $1^{\text {st }}-2^{\text {nd }}$ & 40 \\
\hline This & 3 & $33 \%$ & No & $1^{\text {st }}-4^{\text {th }}$ & 30 \\
\hline This & 5 & $30 \%$ & No & $1^{\text {st }}-3^{\text {rd }}$ & 45 \\
\hline
\end{tabular}

(a) Filter A of that reference.

(b) Refers to the percentage of the length of the conventional counterpart which is reduced.

with 30-dB suppression up to the sixth spurious band, but dimensions are not reduced in such filters. In [57], a method to suppress only the first spurious band in coupled line bandpass filters was proposed. Such technique consists of etching a floating ground-plane conductor, which effectively equalizes the even- and odd-mode phase velocities of the microstrip coupled lines. However, this approach does not represent any advantage in terms of size reduction (although it efficiently suppresses the first harmonic band with a measured rejection level of roughly $35 \mathrm{~dB}$ ).

Other strategies to reduce size and achieve spurious cancellation in coupled line bandpass filters are based on reactive loading [58]-[62]. However, in these techniques, essentially the coupled lines (either open-ended or with grounded cross ports) are either inductively or capacitively loaded in the terminations. Conversely, the strategy proposed in this paper is based on the theory of periodic structures, and coupled line sections with one or two capacitive stages are combined to achieve efficient spurious rejection, as has been justified previously. In the filter reported in [58], 25\% size reduction and a rejection level of better than $47 \mathrm{~dB}$ until the second spurious band $\left(3 f_{0}\right)$ are achieved. In [61], a coupledline bandpass filter with $40-\mathrm{dB}$ suppression until $6.6 f_{0}$ is

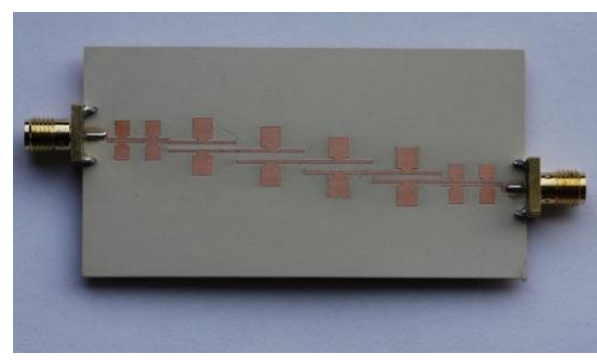

(a)

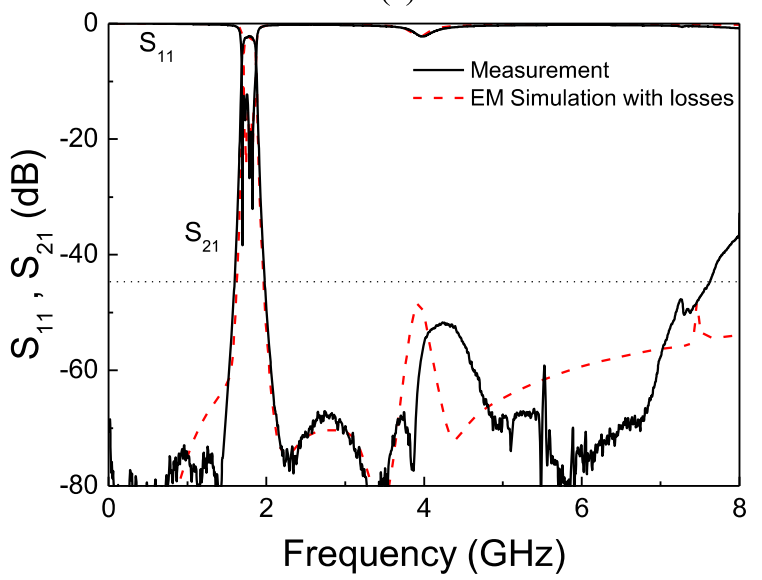

(b)

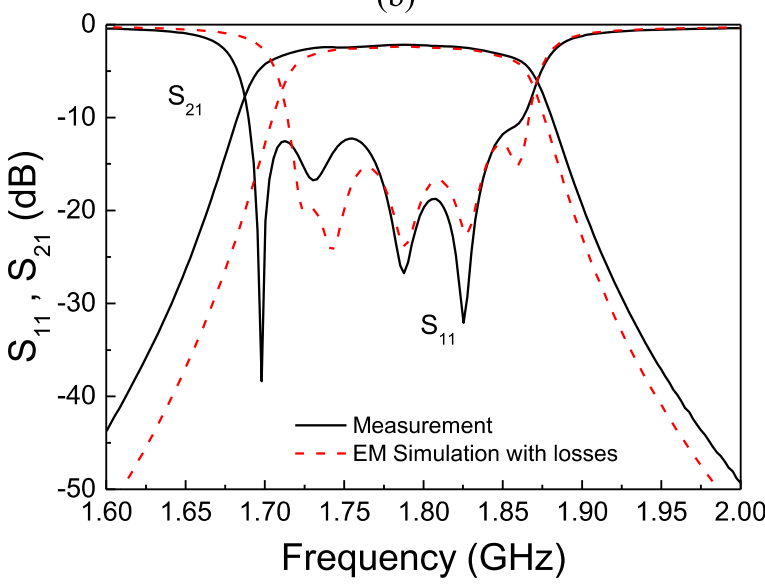

(c)

Fig. 12. (a) Fabricated fifth-order CLCL bandpass filter. (b) Frequency response. (c) Zoomed-in view of the passband.

reported. Such filter is short at the expense of meandering the coupled lines. In [62], a filter with extremely small size (roughly $90 \%$ size reduction compared with the conventional one) is reported, but a rejection level better than $40 \mathrm{~dB}$ is achieved only up to the second spurious band. Other filters reported in [62] exhibit a good balance between size and spurious suppression, but these filters incorporate lumped elements in their designs. A comparison of several coupledline bandpass filters in terms of size reduction and spurious suppression capability is given in Table IX.

In the filters reported in this paper, a good balance between size reduction and spurious suppression is achieved, and filter design is simple since it is based on an automated approach. In particular, the fifth-order filter of Section IV-B exhibits a rejection level better than $45 \mathrm{~dB}$ up to at least $4 f_{0}$. In addition, 
lumped elements are not incorporated in the proposed design approach, which eases fabrication and reduces costs. Note, however, that if lumped elements are allowed in the design, larger capacitance values can be achieved, providing smaller phase velocities and further size reduction.

\section{CONCLUSION}

In conclusion, CLCL bandpass filters and a systematic design approach for such filters, based on ASM optimization, have been proposed for the first time in this paper. Compared with the conventional counterparts, the proposed filters are smaller and spurious free up to higher frequencies, due to the slow-wave effect and stopband functionality introduced by the loading capacitances (in practice, implemented by means of square patches). A model of the CLCL sections, useful for design purposes, i.e., for the determination of the seeding layout necessary to iterate the ASM process, has been proposed. Specifically, from filter specifications and the required slow-wave ratio (directly related to the miniaturization factor), the model provides the even and odd mode characteristic impedances of the host coupled lines, their electrical length, and the value of the loading capacitances, for each coupledline section. From these values, the first (seeding) layout of each CLCL can be inferred using analytical formulas or a transmission-line calculator. Importantly, it has been demonstrated that the number of capacitive unit cells (or pair of patches) of the coupled-line sections cannot be arbitrary. Indeed, to efficiently suppress the first spurious band, it is necessary that at least one coupled-line section is implemented by a single unit cell, and spurious suppression up to very high frequencies can be achieved by combining coupled-line sections with different numbers of unit cells (single and two unit cells in the reported example).

In order to simplify the optimization process of the coupled line sections, the ASM has been divided in two ASM subprocesses. One of them devoted to determine the dimensions (side length) of the patch capacitances; the second one (using these patch dimensions), focused on the automated determination of the layout of each patch-loaded coupled line section. The two illustrative synthesis examples, a third-order and a fifth-order bandpass filter, reported in this paper, have demonstrated the effectiveness of the proposed ASM algorithm to unattendedly determine the layouts of the different coupled lines. By cascading such designed coupled line sections, it has been found that the filter response satisfies the specifications to a good approximation. Moreover, filter size has been reduced to $67 \%$ and $70 \%$ of the size of the ordinary coupled line bandpass filter, for the third-order and fifth-order filters, respectively, and the first spurious bands (at least up to the fourth one and to the third one for the third-order and fifthorder filters, respectively) are efficiently rejected.

\section{REFERENCES}

[1] F. Martín, Artificial Transmission Lines for RF and Microwave Applications. Hoboken, NJ, USA: Wiley, 2015.

[2] E. Yablonovitch, "Photonic band-gap structures," J. Opt. Soc. Amer. B, vol. 10, no. 2, pp. 283-295, 1993.

[3] J. D. Joannopoulos, R. D. Meade, and J. N. Winn, Photonic Crystals. Molding the Flow of Light. Princeton, NJ, USA: Princeton Univ., 1995.
[4] T. Lopetegi et al., "New microstrip 'wiggly-line' filters with spurious passband suppression," IEEE Trans. Microw. Theory Techn., vol. 49, no. 9 , pp. 1593-1598, Sep. 2001.

[5] I. Arnedo et al., "Analytical solution for the design of planar electromagnetic bandgap structures with spurious-free frequency response," Microw. Opt. Technol. Lett., vol. 54, no. 4, pp. 956-960, 2012.

[6] V. Radisic, Y. Qian, R. Coccioli, and T. Itoh, "Novel 2-D photonic bandgap structure for microstrip lines," IEEE Microw. Guided Wave Lett., vol. 8, no. 2, pp. 69-71, Feb. 1998.

[7] F. Falcone, T. Lopetegi, and M. Sorolla, "1-D and 2-D photonic bandgap microstrip structures," Microw. Opt. Technol. Lett., vol. 22, no. 6, pp. 411-412, 1999.

[8] M. A. G. Laso, T. Lopetegi, M. J. Erro, D. Benito, M. J. Garde, and M. Sorolla, "Novel wideband photonic bandgap microstrip structures," Microw. Opt. Technol. Lett., vol. 24, no. 5, pp. 357-360, 2000.

[9] F. Falcone, T. Lopetegi, M. Irisarri, M. A. G. Laso, M. J. Erro, and M. Sorolla, "Compact photonic bandgap microstrip structures," Microw. Opt. Technol. Lett., vol. 23, no. 4, pp. 233-236, 1999.

[10] M. A. G. Laso, T. Lopetegi, M. J. Erro, D. Benito, M. J. Garde, and M. Sorolla, "Multiple-frequency-tuned photonic bandgap microstrip structures," IEEE Microw. Guided Wave Lett., vol. 10, no. 6, pp. 220-222, Jun. 2000.

[11] V. V. Shevchenko, Continuous Transitions in Open Waveguides. Boulder, CO, USA: Golem, 1971.

[12] B. Z. Katsenelenbaum L. Mercader, M. Pereyaslavets, M. Sorolla, and M. Thumm, Theory of Nonuniform Waveguides-The Crosssection Method (IEE Electromagnetic Waves Series). London, U.K.: IEE Press, 1998.

[13] T. Lopetegi, "Photonic band gap structures in microstrip technology: Study using the coupled mode formalism and applications," $\mathrm{Ph}$.D. dissertation, Univ. Pública de Navarra (UPNA), Pamplona, Spain, 2002.

[14] T. Lopetegi et al., "Microstrip 'wiggly-line' bandpass filters with multispurious rejection," IEEE Microw. Wireless Compon. Lett., vol. 14, no. 11 , pp. 531-533, Nov. 2004

[15] A. Gorur, "A novel coplanar slow-wave structure," IEEE Microw. Guided Wave Lett., vol. 4, no. 3, pp. 86-88, Mar. 1994.

[16] A. Gorur, C. Karpuz, and M. Alkan, "Characteristics of periodically loaded CPW structures," IEEE Microw. Guided Wave Lett., vol. 8, no. 8, pp. 278-280, Aug. 1998.

[17] F. Martín et al., "Compact spurious free CPW bandpass filters based on electromagnetic bandgap structures," Microw. Opt. Technol. Lett., vol. 40, no. 2, pp. 146-148, 2004.

[18] J. Garcia-Garcia, J. Bonache, and F. Martín, "Application of electromagnetic bandgaps to the design of ultra-wide bandpass filters with good out-of-band performance," IEEE Trans. Microw. Theory Techn., vol. 54, no. 12 , pp. 4136-4140, Dec. 2006

[19] F. Martin, F. Falcone, J. Bonache, T. Lopetegi, M. A. G. Laso, and M. Sorolla, "New periodic-loaded electromagnetic bandgap coplanar waveguide with complete spurious passband suppression," IEEE Microw. Wireless Compon. Lett., vol. 12, no. 11, pp. 435-437, Nov. 2002.

[20] F. Martín, F. Falcone, J. Bonache, T. Lopetegi, M. A. G. Laso, and M. Sorolla, "Analysis of the reflection properties in electromagnetic bandgap coplanar waveguides loaded with reactive elements," Prog. Electromagn. Res., vol. 42, pp. 27-48, 2003, doi: 10.2528/PIER03022504

[21] F. Martín, F. Falcone, J. Bonache, M. A. G. Laso, T. Lopetegi, and M. Sorolla, "New CPW low-pass filter based on a slow wave structure," Microw. Opt. Technol. Lett., vol. 38, no. 3, pp. 190-193, 2003.

[22] F. Martín, F. Falcone, J. Bonache, T. Lopetegi, M. A. G. Laso, and M. Sorolla, "Dual electromagnetic bandgap CPW structures for filter applications," IEEE Microw. Wireless Compon. Lett., vol. 13, no. 9, pp. 393-395, Sep. 2003

[23] K. W. Eccleston and S. H. M. Ong, "Compact planar microstripline branch-line and rat-race couplers," IEEE Trans. Microw. Theory Techn., vol. 51, no. 10 , pp. 2119-2125, Oct. 2003.

[24] L. Zhu, "Guided-wave characteristics of periodic microstrip lines with inductive loading: Slow-wave and bandstop behaviors," Microw. Opt. Technol. Lett., vol. 41, no. 2, pp. 77-79, 2004.

[25] F.-R. Yang, K.-P. Ma, Y. Qian, and T. Itoh, "A uniplanar compact photonic-bandgap (UC-PBG) structure and its applications for microwave circuit," IEEE Trans. Microw. Theory Techn., vol. 47, no. 8, pp. 1509-1514, Aug. 1999.

[26] J. Sor, Y. Qian, and T. Itoh, "Miniature low-loss CPW periodic structures for filter applications," IEEE Trans. Microw. Theory Techn., vol. 49 , no. 12 , pp. 2336-2341, Dec. 2001 
[27] D. Nesic, "A new type of slow-wave 1-D PBG microstrip structure without etching in the ground plane for filter and other applications," Microw. Opt. Technol. Lett., vol. 33, no. 6, pp. 440-443, 2002.

[28] S.-G. Mao and M.-Y. Chen, "A novel periodic electromagnetic bandgap structure for finite-width conductor-backed coplanar waveguides," IEEE Microw. Wireless Compon. Lett., vol. 11, no. 6, pp. 261-263, Jun. 2001

[29] S.-G. Mao, C.-M. Chen, and D.-C. Chang, "Modeling of slow-wave EBG structure for printed-bowtie antenna array," IEEE Antennas Wireles. Propag. Lett., vol. 1, no. 1, pp. 124-127, 2002.

[30] C. Zhou and H. Y. D. Yang, "Design considerations of miniaturized leas dispersive periodic slow-wave structures," IEEE Trans. Microw. Theory Techn., vol. 56, no. 2, pp. 467-474, Feb. 2008.

[31] D. Kaddour et al., "High-Q slow-wave coplanar transmission lines on $0.35 \mu \mathrm{m}$ CMOS process," IEEE Microw. Wireless Compon. Lett., vol. 19 , no. 9, pp. 542-544, Sep. 2009.

[32] T. LaRocca, J. Y. C. Liu, and M. C. F. Chang, "60 GHz CMOS amplifiers using transformer-coupling and artificial dielectric differential transmission lines for compact design," IEEE J. Solid-State Circuits, vol. 44, no. 5, pp. 1425-1435, May 2009.

[33] J. J. Lee and C. S. Park, "A slow-wave microstrip line with a high-Q and a high dielectric constant for millimeter-wave CMOS application," IEEE Microw. Wireless Compon. Lett., vol. 20, no. 7, pp. 381-383, Jul. 2010

[34] A.-L. Franc et al., "Impact of technology dispersion on slow-wave high performance shielded CPW transmission lines characteristics," Microw. Opt. Technol. Lett., vol. 52, pp. 2786-2789, Dec. 2010.

[35] M. A. Aziz et al., "Slow-wave high-Q coplanar striplines in CMOS technology and their RLCG model," Microw. Opt. Technol. Lett., vol. 54, no. 3, pp. 650-654, 2012

[36] J. G. Lee et al., "Compact semilumped bandstop resonators using the capacitively loaded parallel-coupled microstrip line," Microw. Opt Technol. Lett., vol. 49, no. 2, pp. 367-369, Feb. 2007.

[37] L. Li, F. Xu, K. Wu, S. Delprat, J. Ho, and M. Chaker, "Slow-wave line coupler with interdigital capacitor loading," IEEE Trans. Microw. Theory Techn., vol. 55, no. 11, pp. 2427-2433, Nov. 2007

[38] C. I. Shie, J. C. Cheng, S. C. Chou, and Y. C. Chiang, "Transdirectional coupled-line couplers implemented by periodical shunt capacitors," IEEE Trans. Microw. Theory Techn., vol. 57, no. 12, pp. 2981-2988, Dec. 2009

[39] H. Liu, S. J. Fang, Z. Wang, and Y. Zhou, "Miniaturization of trans-directional coupled line couplers using series inductors," Prog Electromagn. Res. C, vol. 46, pp. 171-177, 2014, doi: 10.2528/PIERC13122201.

[40] J. Selga et al., "Size reduction and spurious suppression in microstrip coupled line bandpass filters by means of capacitive electromagnetic bandgaps," in IEEE MTT-S Int. Microw. Symp. Dig., San Francisco, CA, USA, May 2016, pp. 1-4.

[41] J. W. Bandler, R. M. Biernacki, S. H. Chen, P. A. Grobelny, and R. H. Hemmers, "Space mapping technique for electromagnetic optimization," IEEE Trans. Microw. Theory Techn., vol. 42, no. 12 pp. 2536-2544, Dec. 1994.

[42] J. W. Bandler, R. M. Biernacki, S. H. Chen, R. H. Hemmers, and K. Madsen, "Electromagnetic optimization exploiting aggressive space mapping," IEEE Trans. Microw. Theory Techn., vol. 43, no. 12 pp. 2874-2882, Dec. 1995.

[43] M. H. Bakr, J. W. Bandler, K. Madsen, J. E. Rayas-Sánchez, and J. Søndergaard, "Space-mapping optimization of microwave circuits exploiting surrogate models," IEEE Trans. Microw. Theory Techn. vol. 43, no. 12, pp. 2297-2306, Dec. 2000.

[44] J. W. Bandler, Q. S. Cheng, D. M. Hailu, and K. N. Nikolova, "A space-mapping design framework," IEEE Trans. Microw. Theory Techn., vol. 53, no. 11, pp. 2601-2610, Nov. 2004.

[45] J. V. M. Ros et al., "Fast automated design of waveguide filters using aggressive space mapping with a new segmentation strategy and a hybrid optimization algorithm," IEEE Trans. Microw. Theory Techn., vol. 53, no. 4, pp. 1130-1142, Apr. 2005

[46] S. Koziel, Q. S. Cheng, and J. W. Bandler, "Space mapping," IEEE Microw. Mag., vol. 9, no. 6, pp. 105-122, Dec. 2008.

[47] Q. S. Cheng, J. C. Rautio, J. W. Bandler, and S. Koziel, "Progress in simulator-based tuning-The art of tuning space mapping [application notes]," IEEE Microw. Mag., vol. 11, no. 4, pp. 96-110, Jun. 2010

[48] D. M. Pozar, Microwave Engineering. Reading, MA, USA: AddisonWesley, 1990.

[49] W. R. Eisenstadt, Microwave Differential Circuit Design Using MixedMode S-Parameters. Norwood, MA, USA: Artech House, 2006.
[50] M. Orellana, J. Selga, M. Sans, A. Rodríguez, V. Boria, and F. Martín, "Synthesis of slow-wave structures based on capacitive-loaded lines through aggressive space mapping (ASM)," Int. J. RF Microw. Comput.Aided Eng., vol. 25, no. 7, pp. 629-638, Sep. 2015

[51] J. Selga, A. Rodríguez, M. Gil, J. Carbonell, V. E. Boria, and F. Martín, "Synthesis of planar microwave circuits through aggressive space mapping using commercially available software packages," Int. J. RF Microw. Comput.-Aided Eng., vol. 20, no. 5, pp. 527-534, Sep. 2010.

[52] J. Selga, A. Rodríguez, V. E. Boria, and F. Martín, "Synthesis of split rings based artificial transmission lines through a new two-step, fast converging, and robust aggressive space mapping (ASM) algorithm," IEEE Trans. Microw. Theory Techn., vol. 61, no. 6, pp. 2295-2308, Jun. 2013.

[53] M. Sans, J. Selga, A. Rodríguez, J. Bonache, V. E. Boria, and F. Martín, "Design of planar wideband bandpass filters from specifications using a two-step aggressive space mapping (ASM) optimization algorithm," IEEE Trans. Microw. Theory Techn., vol. 62, no. 12, pp. 3341-3350, Dec. 2014.

[54] M. Sans et al., "Automated design of common-mode suppressed balanced wideband bandpass filters by means of agressive space mapping (ASM)," IEEE Trans. Microw. Theory Techn., vol. 63, no. 12, pp. 3896-3908, Dec. 2015.

[55] J. Bonache, M. Gil, I. Gil, J. Garcia-García, and F. Martín, "On the electrical characteristics of complementary metamaterial resonators," IEEE Microw. Wireless Compon. Lett., vol. 16, no. 10, pp. 543-545, Oct. 2006.

[56] F. Aznar et al., "Characterization of miniaturized metamaterial resonators coupled to planar transmission lines through parameter extraction," J. Appl. Phys., vol. 104, no. 11, p. 114501, Dec. 2008.

[57] M. C. Velazquez-Ahumada, J. Martel, and F. Medina, "Parallel coupled microstrip filters with floating ground-plane conductor for spuriousband suppression," IEEE Trans. Microw. Theory Techn., vol. 53, no. 5, pp. 1823-1828, May 2005.

[58] P. Cheong, S.-W. Fok, and K.-W. Tam, "Miniaturized parallel coupledline bandpass filter with spurious-response suppression," IEEE Trans. Microw. Theory Techn., vol. 53, no. 5, pp. 1810-1816, May 2005.

[59] S.-S. Myoung and J.-G. Yook, "Miniaturisation and harmonic suppression method of parallel coupled-line filters using lumped capacitors and grounding," Electron. Lett., vol. 41, pp. 849-851, Jul. 2005.

[60] S.-S. Myoung, Y. Lee, and J.-G. Yook, "Bandwidth-compensation method for miniaturized parallel coupled-line filters," IEEE Trans. Microw. Theory Techn., vol. 55, no. 7, pp. 1531-1538, Jul. 2007.

[61] S. Lee and Y. Lee, "Generalized miniaturization method for coupledline bandpass filters by reactive loading," IEEE Trans. Microw. Theory Techn., vol. 58, no. 9, pp. 2383-2391, Sep. 2010.

[62] J.-H. Park, S. Lee, and Y. Lee, "Extremely miniaturized bandpass filters based on asymmetric coupled lines with equal reactance," IEEE Trans. Microw. Theory Techn., vol. 60, no. 2, pp. 261-269, Feb. 2012

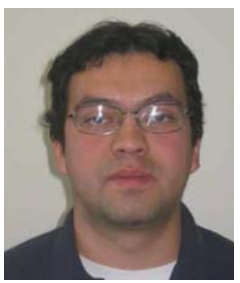

Marco Orellana was born in San José, Costa Rica, in 1985. He received the Licentiate degree in electrical engineering from the Universidad de Costa Rica (UCR), San José, in 2009, and the master's degree in micro and nanoelectronics engineering from the Universitat Autònoma de Barcelona, Barcelona, Spain, in 2011, with the support of the Erasmus Mundus Program and the UCR, where he is currently involved in his doctoral thesis focusing on the automation of metamaterial-based structures design.

He was an Instructor with the School of Electrical Engineering, UCR.

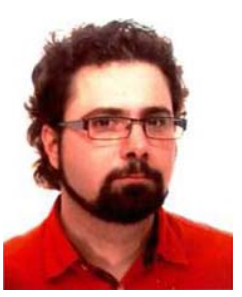

Jordi Selga (S'11-M'14) was born in Barcelona, Spain, in 1982. He received the B.S. degree in telecommunications engineering-electronic systems and M.S. and Ph.D. degrees in electronics engineering from the Universitat Autònoma de Barcelona (UAB), Barcelona, in 2006, 2008, and 2013, respectively.

$\mathrm{He}$ is currently with $\mathrm{UAB}$, where he is involved in subjects related to metamaterials, CAD design of microwave devices, electromagnetic optimization methods, and automated synthesis of planar

microwave components.

Prof. Selga has been a member of CIMITEC-UAB, a research center on Metamaterials supported by TECNIO (Catalan Government) since 2008. He was the recipient of the National Research Fellowship from the Formación De Profesorado Universitario Program of the Education and Science Ministry (Reference AP2008-4707). 


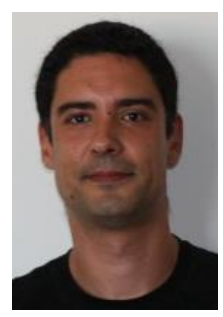

Paris Vélez (S'10-M'14) was born in Barcelona, Spain, in 1982. He received the degree in telecommunications engineering (with a specialization in electronics), electronics engineering degree, and $\mathrm{Ph} . \mathrm{D}$. degree in electronics engineering from the Universitat Autònoma de Barcelona, Barcelona, in 2008, 2010, and 2014, respectively. His Ph.D. thesis was entitled "Common mode suppression differential microwave circuits based on metamaterial concepts and semilumped resonators."

His current research interests include the miniaturization of passive circuits RF-/microwave-based metamaterials

Dr. Vélez was awarded the Predoctoral Teaching and Research Fellowship by the Spanish Government from 2011 to 2014. He is currently a reviewer for the IEEE TRANSACTIONS ON MicRowave THEORY AND TECHNIQUES and other journals.

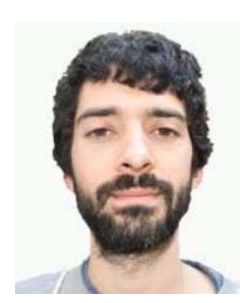

Marc Sans was born in Terrassa, Spain, in 1982 $\mathrm{He}$ received the B.S. degree in telecommunications engineering-electronic systems, M.S. degree in telecommunications engineering, and M.S. degree in electronics engineering from the Universitat Autònoma de Barcelona (UAB), Barcelona, Spain, in 2006, 2008, and 2013, respectively, where he has been pursuing the Ph.D. degree with CIMITEC$\mathrm{UAB}$, focusing on the synthesis of microwave devices based on electromagnetic optimization techniques.

In 2008, he joined Sony-FTVE as an RF Engineer, where he developed the RF stage of TV receivers. In 2010, he joined Mier Comunicaciones S.A. Barcelona, to carry out the design of passive and active devices for VHF-UHF broadcasting units.

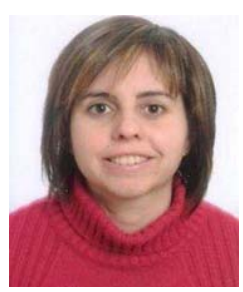

Ana Rodríguez (S'10-M'14) was born in Lugo, Spain. She received the telecommunications engineering degree from the Universidade de Vigo, Vigo, Spain, in 2008, and Master en Tecnología, Sistemas y Redes de Comunicaciones and Ph.D. degree from the Universitat Politècnica de València (UPV), Valencia, Spain, in 2010 and 2014, respectively.

As a student, she participated in the Erasmus exchange program, developing the master's thesis at the University of Oulu, Oulu, Finland. Since 2008, she has been with the Institute of Telecommunications and Multimedia Applications, which is part of the scientific park, UPV. Her current research interests include CAD design of microwave devices, electromagnetic optimization methods, and metamaterials.

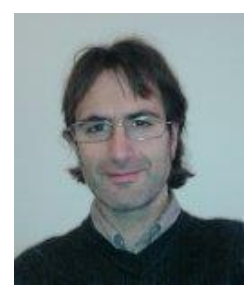

Jordi Bonache (S'05-M'07) was born in Barcelona, Spain, in 1976. He received the physics and electronics engineering degrees and Ph.D. degree in elec tronics engineering from the Universitat Autònom de Barcelona (UAB), Barcelona, in 1999, 2001, and 2007, respectively.

In 2000, he joined the High Energy Physics Institute, Barcelona, where he was involved with the design and implementation of the control and monitoring system of the MAGIC telescope. In 2001, he joined the Department of Electronics Engineering, UAB, where he is currently an Associate Professor. From 2006 to 2009, he was an Executive Manager with CIMITEC, Drachten, The Netherlands, where he currently leads the research in RFID and antennas. His current research interests include active and passive microwave devices, metamaterials, antennas, and RFID.

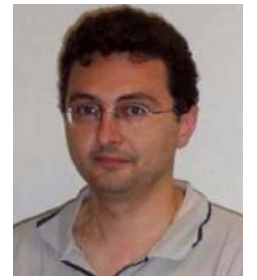

Vicente E. Boria (S'91-A'99-SM'02) was born in Valencia, Spain, in 1970 . He received the Ingeniero de Telecomunicación (with First Class Hons.) degree and Doctor Ingeniero de Telecomunicación degree from the Universidad Politécnica de Valencia, Valencia, in 1993 and 1997, respectively.

In 1993, he joined the Departamento de Comunicaciones, Universidad Politécnica de Valencia, where he has been a Full Professor since 2003. From 1995 to 1996 , he held a Spanish Trainee position with the European Space Research and Technology Center, European Space Agency, Noordwijk, The Netherlands, where he was involved in the area of electromagnetic analysis and design of passive waveguide devices. He has authored or coauthored ten chapters in technical textbooks, 135 papers in refereed international technical journals, and over 180 papers in international conference proceedings. His current research interests include the analysis and automated design of passive components, left-handed and periodic structures, as well as the simulation and measurement of power effects in passive waveguide systems.

Dr. Boria is a member of the Technical Committees of the IEEE Microwave Theory and Techniques Society (MTT-S) International Microwave Symposium and the European Microwave Conference, and has been a member of the IEEE MTT-S and the IEEE Antennas and Propagation Society since 1992. $\mathrm{He}$ is currently a reviewer for the IEEE TRANSACTIONS ON MICROWAVE THEORY AND TECHNIQUES and IET Microwaves, Antennas, and Propagation and serves as an Associate Editor of the IEEE MICROWAVE AND WIRELESS COMPONENTS LETTERS and IET Electronics Letters.

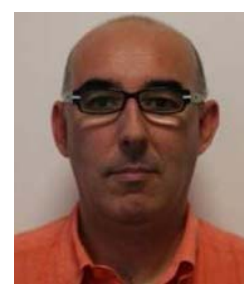

Ferran Martín (M'04-SM'08-F'12) was born in Barakaldo, Spain, in 1965. He received the B.S. degree in physics and Ph.D. degree from the Universitat Autònoma de Barcelona (UAB), Barcelona, Spain, in 1988 and 1992, respectively.

From 1994 to 2006, he was an Associate Professor of electronics with the Departament d'Enginyeria Electrònica, UAB, and since 2007, he has been a Full Professor of electronics. He is currently the Head of the Microwave Engineering, Metamaterials, and Antennas Group, UAB, and the Director of CIMITEC, a research Center on Metamaterials supported by TECNIO, Generalitat de Catalunya, Barcelona. He has authored or coauthored over 500 technical conference papers, letters, journal papers, and book chapters. He coauthored a book on metamaterials entitled Metamaterials with Negative Parameters: Theory, Design, and Microwave Applications (Wiley, 2008) and an author of the book Artificial Transmission Lines for $R F$ and Microwave Applications (Wiley, 2015), and he has supervised 15 Ph.D. students $\mathrm{He}$ held several patents on metamaterials and has headed several development contracts. He is involved in different research activities including modeling and simulation of electron devices for high-frequency applications, millimeterwave and terahertz generation systems, and the application of electromagnetic bandgaps to microwave and millimeter-wave circuits. His current research interests include metamaterials and their application to the miniaturization and optimization of microwave circuits and antennas

Prof. Martín is Fellow of the IET $\mathrm{He}$ is a member of the IEEE Microwave Theory and Techniques Society (MTT-S), the Editorial Board of IET Microwaves, Antennas, and Propagation and the International Journal of $R F$ and Microwave Computer-Aided Engineering. He is also a member of the Technical Committees of the European Microwave Conference and the International Congress on Advanced Electromagnetic Materials in Microwaves and Optics (Metamaterials). Among his distinctions, he was a recipient of the 2006 Duran Farell Prize for Technological Research and two ICREA ACADEMIA Awards (calls 2008 and 2013), and holds the Parc de Recerca $U A B$-Santander Technology Transfer Chair. He has served as a Guest Editor of three Special Issues on Metamaterials in three international journals. $\mathrm{He}$ has organized several international events related to metamaterials, including Workshops at the IEEE International Microwave Symposium (2005 and 2007), the European Microwave Conference (2009), and the Fifth International Congress on Advanced Electromagnetic Materials in Microwaves and Optics (Metamaterials 2011), where he has served as the Chair of the Local Organizing Committee. $\mathrm{He}$ is currently a reviewer for the IEEE TRANSACTIONS ON Microwave THEORY AND TeCHNIQUES and the IEEE MicrowaVE AND WIRELESS COMPONENTS LETTERS, among many other journals. 